\title{
LINGUISTIQUE
}

\author{
MARZENA BLACHOWSKA
}

Universitê Adam Mickiewicz à Poznań

\section{SAVOIR ARGUMENTER: APPROCHE INTERACTIVE}

\begin{abstract}
A b st r a ct. Blachowska Marzena, Savoir argumenter: approche interactive [To know how to write and understand argumentative texts: An interactive approach]. Studia Romanica Posnaniensia, Adam Mickiewicz University Press, Poznan, vol. XXIX: 2003, pp. 89-127, ISBN 83-232-1232-5, ISSN 0137-2475.
\end{abstract}

In this article the author deals with the question of teaching the skill of writing and understanding of argumentative texts in French as a foreign language at the advanced level. The author suggests that the interactive approach be adopted in teaching argumentative writing and understanding and characterizes its goals and principles, then the author presents gradation of the content of teaching. Particular stages of gradation are illustrated didactic sequences.

Le présent travail entreprend de présenter les principes et les contenus de l'approche interactive de l'enseignement/apprentissage de l'argumentation écrite adaptée aux besoins des étudiants des philologies romanes et des collèges de langues. Si ceux-ci réussissent, tant bien que mal, à comprendre/rédiger une description, une narration et des paragraphes argumentatifs (justificatifs, réfutatifs et contre-argumentatifs), ils restent perplexes face à des textes de type argumentatif (en particulier face à la dissertation). Dans les matériels consacrés à l'enseignement/ apprentissage de l'argumentation écrite en FLE, l'accent est mis généralement:

- sur la visée pragmatique des textes et ses marques (non)linguistiques;

- sur les planifications des textes et leurs marques (non)linguistiques.

En d'autres termes, l'argumentation est d'habitude considérée comme une propriété du discours, ce qui ressort de la théorie de l'argumentation dans le discours (Moeschler 1984) ou bien comme un type de texte particulier, ce qui relève de la théorie de l'argumentation dans la langue (Ducrot 1980, 1984) et de ses racines rhétoriques (voir Aristotes, Perelman in Declercq 1993). Or, les étudiants 
polonais ont le plus de problème, à la lecture et à l'écriture, avec la macrostructure des types de textes (autrement dit, leur sens global) et avec leurs schémas formels.

Ainsi, afin de développer la capacité à comprendre/appliquer une attitude polémique à l'égard des faits/des savoirs énoncés, nous proposons:

- d'insister - dès le début du cycle de l'enseignement supérieur - sur le développement de la capacité à comprendre la/les visée(s) pragmatique(s) (autrement dit, l'orientation argumentative globale ou l'argumentativité) et le/les schéma(s) formel(s) des types de textes;

- de développer la capacité à comprendre la visée pragmatiques des types de textes en interaction avec la capacité à produire des types de textes cohésifs et cohérents.

Il reste à préciser que pour réaliser notre objectif, nous nous appuyons, d'une part sur une typologie séquentielle inspirée de celle de J.-M. Adam (1997), d'autre part sur un classement des modalités d'attitude du sujet énonçant à l'égard des faits/des savoirs énoncés conçu, entre autres, à partir des travaux sur l'énonciation d'O. Ducrot $(1980,1984)$ de J.-P. Bronckart (1985) et de K. Kerbrat-Orecchioni (1980). Nous distinguons donc quatre schémas prototypes relevant de la superstructure monologale, à savoir les schémas:

- descriptif se caractérisant par les arrangements textuels d'ordre tabulaire/hiérarchique, ce qui se manifeste dans le texte par le recours aux anaphores, à la co-référence et aux organisateurs énumératifs;

- narratif se développant à partir des arrangements textuels chrono-logiques (c'est-à-dire temporels et logiques), ce qui se traduit par le recours aux marques de la localisation temporelle, aux organisateurs temporels et aux connecteurs (avant tout ceux qui expriment des rapports de cause, de conséquence et de finalité);

- expositif-explicatif combinant les arrangements textuels d'ordre tabulaire/hiérarchique et logique (appliqués à des objets abstraits ou à des idées et aux prémisses vraies);

- argumentatif se développant à partir des prémisses vraisemblables et selon l'ordre logique ou bien tabulaire/hiérarchique et logique.

Se combinant sur le plan textuel, les séquences prototypes donnent lieu aux quatre types de textes à structure séquentielle hétérogène dont chacun cherche, bien entendu, l'acceptabilité émotionnelle et/ou intellectuelle du lecteur-cible. Compte tenu de leur hétérogénéité séquentielle et de leur visée pragmatique, nous parlons des types de textes argumentés à orientation descriptive, narrative, expositive-explicative et argumentative.

Pour trouver l'acceptabilité émotionnelle et/ou intellectuelle du lecteur-cible, le sujet énonçant nuance son attitude à l'égard des faits/des savoirs énoncés, ce qui se manifeste à travers le texte par: 
- les rapports entre le sujet énonçant et le cadre spatio-temporel d'énonciation;

- le degré d'assertion (autrement dit, l'évaluation de type vrai/faux/incertain) et d'axiologisation (i.e. l'évaluation de type bon/mauvais) de l'objet du discours;

- le degré d'engagement émotionnel du sujet énonçant dans l'objet de son discours (i.e. l'évaluation affective de l'objet de son discours).

En somme, dans les textes argumentés à orientation descriptive et narrative, le sujet énonçant qualifie/se distancie, dans les textes argumentés à orientation expositive-explicative, il cherche à objectiver et dans les textes argumentés à orientation argumentative, il tâche de prendre position.

Ainsi, afin de développer la capacité à comprendre/appliquer une attitude polémique, nous privilégions le travail sur:

- les types de schémas formels et leur combinaison dans les types de textes particuliers, ce qui contribue à marquer d'une part la spécificité des types de textes, d'autre part les parentés entre les types de textes;

- les modalités d'attitude du sujet énonçant (qualification/distanciation, objectivation et prise de position), ce qui permet de donner un aperçu global des moyens linguistiques (et non-linguistiques) contribuant à spécifier l'acceptabilité visée par le texte (émotionnelle/intellectuelle).

Dans le processus d'enseignement/apprentissage de l'argumentation écrite, le rôle de l'enseignant consiste grosso modo à encourager l'interaction entre l'étudiant avec tout son bagage cognitif (i.e. ses connaissances et ses expériences) et les textes particuliers. L'enseignant devient donc coordinateur du processus d'apprentissage et considère l'étudiant comme un sujet conscient de ses besoins et de ses progrès.

En ce qui concerne le choix du support, nous proposons des séquences didactiques exemplaires conçues à partir d'articles de presse. Il faut pourtant préciser que nous ne traitons pas les textes de presse comme des modèles d'écriture et que le choix du type de discours écrit pour l'enseignement/apprentissage de l'argumentation dépend uniquement de l'enseignant. Ce dernier peut même travailler sur différents types de discours mais tout en tenant compte de leur spécificité.

En somme, le caractère interactif de l'enseignement/apprentissage de l'argumentation écrite se traduit principalement par le choix des contenus (l'interaction entre les types de schémas formels et les modalités d'attitude du sujet énonçant, l'interaction entre les types de textes) et de la démarche (l'interaction entre les capacités de compréhension et de production) ainsi que par la définition des rôles de l'enseignant et de l'étudiant. Une telle pédagogie de l'argumentation facilitera la lecture et l'écriture des textes polémiques par l'étudiant. De plus, elle le préparera indirectement à l'étape consacrée à l'élaboration de la dissertation française. 


\section{LES PRINCIPES DE BASE DE L'APPROCHE INTERACTIVE}

Notre conception des rôles de l'étudiant et de l'enseignant s'inscrit dans la lignée de la tendance actuelle à autonomiser le processus d'apprentissage selon laquelle l'apprenant est responsable de ses progrès et le rôle de l'enseignant consiste à le guider (Rivers 1990: 40). En effet, il nous est difficile d'imaginer un enseignement/apprentissage de l'argumentation qui consisterait à accumuler des savoirs car l'action d'argumenter implique la capacité à entrer en duel avec le réel. Pour y arriver l'étudiant doit savoir agir sur son propre apprentissage. Respectivement, la tâche de l'enseignant n'est pas à transmettre des savoirs mais à stimuler le développement cognitif de l'étudiant.

\subsection{L'ÉTUDIANT: UN SUJET CONSCIENT DE SON APPRENTISSAGE}

L'étudiant conscient de son apprentissage est capable de le gérer ainsi que d'entrer en interaction avec l'enseignant et d'autres étudiants afin d'échanger des savoirs et des points de vue. Ces deux savoir-faire sont particulièrement importants pour son développement cognitif et pour le développement de sa capacité à entrer en duel avec le réel.

\subsubsection{SAVOIR GÉRER SON APPRENTISSAGE}

La compréhension/production des textes argumentés demande à notre étudiant un double effort cognitif. Il doit non seulement acquérir de nouvelles connaissances morpho-syntaxiques, sémantiques, pragmatiques et socio-culturelles, mais aussi savoir les activer:

- lors de la lecture pour reconstruire le schéma formel et définir/interpréter l'attitude du sujet énonçant;

- lors de l'écriture pour produire des textes argumentés cohésifs-cohérents.

Cette tâche s'avère souvent trop difficile pour lui. Se concentrant d'habitude sur la compréhension de nouveaux mots, il perd de vue le sens global du texte. Lecteur habile en langue maternelle, en langue seconde il procède par le déchiffrage et recourt à la traduction comme s'il avait perdu confiance en ses connaissances pragmatiques et socio-culturelles et qu'il ne sache plus y faire appel pour en tirer des inférences. En somme, lors de la lecture, le comportement de l'étudiant semble avoir un caractère hiérarchique: il cherche, en premier lieu, à trouver des liens de surface au niveau de la microstructure du texte mais le coût cognitif de cette opération s'avère souvent beaucoup trop élévé pour qu'il puisse construire, par la suite, une représentation de la macrostructure du texte (donc de son sens global). Cet attachement à la surface du texte peut se manifester à travers ses écrits, par 
exemple dans une tendance à l'hypercorrection au niveau de la microstructure et le manque de cohérence au niveau de la macrostructure.

La question se pose pourquoi l'étudiant s'attarde sur la signification de tout mot inconnu et recourt à la traduction au lieu, par exemple, d'esquiver les difficultés. Abstraction faite du cas où ses connaissances morpho-syntaxiques et sémantiques sont vraiment trop limitées pour qu'il puisse tirer des inférences (par exemple, il bute sur tous les deux ou trois mots), un tel comportement est dû, à notre avis, à ses représentations stéréotypées de la langue et du processus d'enseignement/ apprentissage. Le plus souvent, l'étudiant considère la langue comme code et attache, par la suite, beaucoup d'importance au vocabulaire et aux règles morpho-syntaxiques. Il identifie l'apprentissage avec la réception des savoirs transmis par l'enseignant et les accumule thématiquement (par exemple, selon les matières), ce qui rend difficile leur activation-configuration. Pour savoir entrer en interaction avec les sources de connaissances nouvelles, il faut que l'étudiant commence par prendre conscience de ses habitudes d'apprentissage et qu'il apprenne à gérer ses progrès, ce qui pourra s'effectuer par l'auto-observation et l'auto-évaluation. Comme le constate W. Rivers: «Les apprenants doivent se rendre compte du fait qu'ils sont responsables de leurs propres progrès; ils prendront leur responsabilité plus sérieusement s'ils découvrent et corrigent eux-mêmes leurs propres faiblesses» (Rivers 1990: 48).

De plus, l'auto-observation et l'auto-évaluation permettront à l'étudiant de connaître, dans une certaine mesure, son (ses) styles cognitif(s), c'est-à-dire «...des aptitudes, bien que le fonctionnement cognitif ne soit pas envisagé prioritairement sous l'angle de son efficience, et des traits de personnalité» (Huteau 1987: 8 in M. Perraudeau 1996: 46). Vu leur quantité et leur diversité - M. Huteau identifie une vingtaine de styles cognitifs (Perraudeau 1996: 46) - il lui sera difficile, voire impossible, de le/les définir exactement. Globalement, les styles cognitifs s'attachent:

- à la prise d'informations (la dépendance-indépendance);

- à la perception d'informations (la pensée latérale);

- à la manière de maîtriser des savoirs (l'approche dure-douce, l'accentuation-égalisation, le mode d'évocation visuelle-auditive et la centration-balayage);

- au comportement du sujet en situation d'apprentissage (l'impulsivité versus la réflexion, le contrôle interne versus externe, la consommation versus la production).

Pour conclure, la prise de conscience de ses habitudes d'apprentissage (ses habitudes de lecture) permettra à l'étudiant d'évaluer leur efficacité et de réfléchir sur les possibilités de les modifier. Ceci contribuera, par la suite, à améliorer sa compréhension des textes argumentés et débouchera sur l'amélioration de la qualité de ses écrits. 
Le comportement de l'individu en situation d'interaction diffère d'une part selon son style cognitif, d'autre part selon le rôle social qu'il a à jouer à un moment et à un endroit donnés (par exemple, le rôle de fils à la maison versus le rôle d'étudiant à l'université) et selon son environnement social (c'est-à-dire d'autres individus, leur style cognitif et leur rôle social, par exemple ses amis, ses condisciples, ses parents, ses enseignants, etc.). De plus, il va de soi que les aptitudes et les traits de personnalité de l'individu influencent la qualité de ses représentations du monde (par exemple, un étudiant dépendant du champ aura du mal à porter un regard critique sur qui que se soit et quoi que se soit et ses représentations du monde risqueront de paraître naïves). Ainsi, dans un même groupe, il y aura des étudiants n'éprouvant aucune difficulté à entrer en interaction ni avec leurs collègues ni avec leur enseignant et n'ayant besoin d'aucun encouragement et à l'autre extrémité des étudiants préférant laisser agir leurs collègues.

Néanmoins, l'échange des savoirs, des points de vue et des vécus entre les individus est particulièrement important pour notre pédagogie de l'argumentation écrite car il permet à l'étudiant d'activer/confronter ses connaissances préalables et, par la suite, d'accommoder de nouvelles connaissances. Le caractère polémique de l'argumentation lui demande même de savoir entrer en interaction aussi bien avec l'enseignant qu'avec les autres étudiants et ceci malgré ses difficultés linguistiques et quelquefois à l'encontre de son style cognitif.

La communication avec l'enseignant concerne en premier lieu la réalisation des activités en classe/en dehors de la classe et le processus même d'enseignement/ apprentissage. L'étudiant ne devrait donc pas hésiter:

- à poser des questions pour vérifier la compréhension des consignes;

- à demander des précisions et des explications au sujet du programme et de sa réalisation;

- à évaluer l'efficacité des procédés et des techniques utilisés par l'enseignant;

- à demander des conseils au sujet de son propre apprentissage.

De plus, il va de soi que l'étudiant devrait réagir aux questions et aux explications de l'enseignant. En bref, «l'apprentissage nécessite une confrontation intellectuellement conflictuelle entre apprenant et médiateur (maître ou élève)...» (Perraudeau 1996: 39). Ce principe reste particulièrement important dans le cadre de l'enseignement de l'argumentation qui correspond au développement de la capacité à entrer en duel avec le réel.

Pourtant, ce qui peut rendre difficile cette confrontation intellectuellement conflictuelle, en dehors du niveau linguistique et du style cognitif du sujet étudiant, c'est une différence d'âge trop élevée et une distance cognitive trop importante entre l'étudiant et l'enseignant. Par conséquent, au lieu de questionner le savoir apporté par l'enseignant, l'étudiant l'accepte sans hésitation (Perraudeau 1996: 39). 
Quant à la communication entre les étudiants, elle porte sur la coopération pendant la réalisation des activités en classe/en dehors de la classe ainsi que sur le processus d'apprentissage. La coopération demande aux étudiants de savoir partager les responsabilités, d'échanger les données et de comparer les résultats. La communication au sujet du processus d'apprentissage reste en rapport avec la capacité à gérer son apprentissage et concerne l'échange des stratégies d'apprentissage et l'évaluation du travail effectué.

Ce qui rapproche les étudiants c'est, en premier lieu, leur âge et le niveau cognitif semblable. En revanche, ce qui les différencie et influence la qualité de leurs interactions, ce sont leur style cognitif, leurs intérêts et leur motivation.

\subsubsection{BILAN}

En développant les capacités à gérer son apprentissage et à entrer en interaction avec l'enseignant et les autres étudiants, l'étudiant exerce et accroît ses habiletés générales telles que l'esprit d'observation, le raisonnement logique, la créativité, etc. Ceci contribuera à lui faciliter l'activation/configuration de ses connaissances de faible niveau (typographiques, morpho-syntaxiques et sémantiques) et de haut niveau (pragmatiques et socio-culturelles) aussi bien lors de la lecture que lors de l'écriture des textes argumentés. Ainsi, le développement des habiletés générales conditionne le développement de la capacité à argumenter.

\subsection{L'ENSEIGNANT: UN GUIDE ET UN COORDINATEUR DU PROCESSUS D'APPRENTISSAGE}

Puisque la compréhension/production des textes argumentés nécessite une interaction entre les connaissances préalables de l'étudiant et les sources des connaissances nouvelles, le rôle de l'enseignant ne peut pas consister à transmettre le savoir mais à créer un environnement favorable pour le développement des interactions entre:

- l'étudiant et le texte;

- l'étudiant et l'enseignant;

- les êtudiants;

- l'étudiant et son apprentissage.

1.2.1. DÉVELOPPER L'INTERACTION ENTRE L'ÉTUDIANT ET LE TEXTE LU/ÉCRIT: PRINCIPES ET ATOUTS DE LA LECTURE INTERACTIVE

Lors de la lecture en langue étrangère se concentrant trop sur le déchiffrage des mots et des structures inconnus, l'étudiant a du mal à créer la représentation cognitive du texte. Ceci a un impact sur la qualité de ses écrits: comment peut-il 
produire un texte argumentatif, ou un autre type de texte cohérent sans pouvoir se référer à sa représentation cognitive? Il s'avère donc particulièrement important de développer sa capacité à comprendre des textes pour améliorer sa capacité à rédiger des textes cohésifs-cohérents. Autrement dit, l'objectif de la lecture interactive sera d'amener l'étudiant à se construire des représentations cognitives des textes argumentés en langue étrangère et de le préparer à les activer lors de l'écriture, ce qui reste en rapport direct avec le développement de son savoir métacognitif sur les stratégies cognitives en général et sur les stratégies de lecture en particulier. En bref, l'application de la démarche interactive permettra à l'enseignant d'assurer aussi bien le développement cognitif que le développement métacognitif de l'étudiant. Le principal avantage de la lecture interactive est que la compréhension textuelle est obtenue par paliers et que chacun d'eux a son objectif. Aussi l'étudiant n'est-il pas obligé de comprendre «tout» lors de la première lecture: «L'approche interactive est une méthodologie dans laquelle le lecteur va comprendre le texte en le reliant à ce qu'il connaît déjà (aussi bien sur le plan du thème traité que sur celui de la structure et du genre du texte), en découvrant dans le texte des éléments (...) qui lui permettent de confirmer ses hypothèses et d'atteindre une compréhension parfois partielle» (Cicurel 1991: 43). De plus, la lecture interactive débouche sur des activités d'écriture, ce qui permet de verifier l'engrengement des connaissances nouvelles et de développer la capacité à écrire des textes cohésifs-cohérents.

Quant à l'enseignant, il peut organiser ses cours d'une façon plus souple et plus variée que dans le cas d'un enseignement/apprentissage plus traditionnel de la lecture où la compréhension de textes était d'habitude mesurée par le taux de bonnes réponses fournies par l'étudiant. F. Cicurel (1991: 43) distingue quatre étapes de la lecture interactive:

- l'étape 1 dont l'objectif est d'activer-orienter des connaissances préalables de l'étudiant (l'apprenant) avant la distribution des textes;

- l'étape 2 qui consiste à observer-prélever des marques typographiques et linguistiques, elle a pour objectif de familiariser l'étudiant (l'apprenant) avec le texte et de le faire anticiper sur son schéma formel et l'attitude du sujet énonçant;

- l'étape 3 qui correspond à une lecture-recherche, elle consiste en une sélection d'une ou de plusieurs entrées par lesquelles on accède au sens du texte. Ainsi, la compréhension du texte résulte soit des lectures successives - l'étudiant (l'apprenant) relit le texte en réalisant à chaque fois des consignes différentes - soit de l'échange des informations entre les petits groupes d'étudiants (d'apprenants) dont chacun réalise une consigne différente;

- l'étape 4 a pour objectif de vérifier l'engrangement de nouvelles connaissances. Il est demandé à l'étudiant (l'apprenant) de réagir soit oralement soit par écrit au texte lu.

La réalisation des objectifs en passant par chaque étape de la lecture interactive oblige l'étudiant à rompre avec la lecture linéaire et l'encourage à prélever globalement les mots ou les groupes de mots, à prédire les informations qui vont 
suivre dans le texte et ajuster son fonctionnement cognitif à l'activité réalisée (ou à son projet de lecture). De plus, l'étudiant se trouve dans la nécessité même d'accepter une certaine imprécision, de recourir au contexte du mot ou de l'énoncé, de tirer des inférences, de se référer à ses connaissances préalables et de faire le point par rapport à son intention (projet) de lecture, à ses besoins et à ses intérêts. En bref, cette démarche interactive permet à l'étudiant de prendre conscience des habiletés et des stratégies de lecture en langue maternelle (Cornaire 1991: 38-39).

En outre, chaque étape de la lecture interactive favorise l'application des stratégies mentales différentes, à savoir:

- l'étape 1 demande à l'étudiant de procéder par égalisation;

- l'étape 2 oblige l'étudiant à considérer plusieurs indices simultanément donc de procéder par balayage;

- l'étape 3 privilégie le travail intensif et la démarche par centration;

- l'étape 4 permet de relier la consommation et la production, de valoriser la démarche par accentuation ainsi que de vérifier l'équilibration cognitive de l'étudiant.

La démarche interactive contribue aussi à dédramatiser l'erreur: même si les hypothèses ou les inférences s'avèrent erronées l'étudiant peut les vérifier, ce qui convient aussi bien aux sujets impulsifs qu'aux sujets réflexifs.

Il résulte de ce qui précède que la lecture interactive facilite l'alternance de différentes stratégies mentales et permet de développer chez l'étudiant la capacité à activer/configurer ses connaissances de faible niveau et de haut niveau lors de la lecture afin de construire les représentations cognitives des textes et de produire des textes cohésifs-cohérents. De plus, elle constitue une bonne base pour le développement des interactions entre les étudiants et l'enseignant, ainsi qu'entre l'étudiant et son apprentissage.

\subsubsection{DÉVELOPPER L'INTERACTION ENTRE L'ENSEIGNANT ET LES ÉTUDIANTS}

L'interaction entre l'enseignant et les étudiants ne peut se développer que dans une ambiance de confiance et de bienveillance réciproque. Pour y parvenir l'enseignant doit connaître ses étudiants. Ceci lui permettra d'adapter - dans la mesure du possible - le contenu des cours et les procédés utilisés aux besoins des étudiants donc de diminuer les différences entre leurs styles d'apprentissage et son style d'enseignement, comme le remarque W. Rivers: «Les professeurs de langues doivent connaître les apprenants dans leurs classes (leur âge, leur passé, leurs aspirations, leurs intérêts, leurs buts dans l'apprentissage d'une langue, leur aptitude pour l'acquisition des langues dans un contexte scolaire) et concevoir ensuite des cours de langues qui répondent aux besoins de chaque groupe spécifique» (Rivers 49: 1990). 
Ainsi, avant même de commencer la réalisation du programme, l'enseignant devra effectuer des tests pronostiquant le niveau des connaissances préalables des étudiants, ainsi que des tests permettant de définir leurs représentations de la situation d'enseignement/apprentissage. Ensuite, durant le cursus, l'enseignant devra effectuer des tests diagnostics afin de suivre l'évolution de ses étudiants et de réguler son propre enseignement. De plus, il est important qu'il sache écouter les avis et les propositions des étudiants pour en tirer des conclusions sur leurs besoins.

Pour créer une ambiance amicale, l'enseignant doit développer ses aptitudes à communiquer (Rivers 1990: 51) et à contrôler les émotions. Ce qui est également important c'est l'attitude de l'enseignant face à l'erreur: l'évaluation continue et sommative ne devraient pas être considérées par les étudiants comme une punition mais comme «...un indicateur signalant, ce qui a été vraiment assimilé de telle sorte que cela soit disponible pour un emploi réaliste...» (Rivers 1990: 56). En bref, il faut dédramatiser l'erreur. Pour ce faire trois conditions sont à remplir: premièrement l'évaluation doit faire partie du cours, deuxièmement la grille d'évaluation doit être connue et claire pour l'étudiant et troisièmement l'enseignant doit encourager les étudiants à analyser leurs erreurs et à s'évaluer.

En résumé, pour encourager les étudiants à prendre la parole, ce qui nous semble particulièment important pour le développement de la capacité à argumenter, l'enseignant doit connaître leurs attentes et leurs besoins, savoir écouter leurs avis et leurs propositions, contrôler ses émotions et dédramatiser l'erreur.

\subsubsection{DÉVELOPPER L'INTERACTION ENTRE LES ÉTUDIANTS}

Pour assurer l'échange des savoirs et la coopération entre les étudiants il est indispensable de varier les modalités de travail et les procédés utilisés: la domination d'un mode de travail ou d'un procédé particuliers ne peut, en fait, activer qu'une partie des étudiants. Ainsi, le travail individuel devrait se conjuguer avec le travail en tandem et en petits groupes. De plus, il s'avère important de proposer des activités qui demanderaient à l'étudiant de recourir aux stratégies mentales différentes, par exemple l'analogie versus la différence, l'inférence versus la déduction, la synthèse versus l'analyse (voir aussi 1.2.1).

En bref, l'alternance des démarches permet non seulement d'activer tous les étudiants du groupe, quel que soit leur style d'apprentissage, mais aussi d'enrichir dans la mesure du possible - leurs stratégies mentales (bien entendu à condition qu'ils soient conscients de leur propre apprentissage).

\subsubsection{DÉVELOPPER L'INTERACTION ENTRE L'ÉTUDIANT ET SON APPRENTISSAGE}

L'étudiant qui a du mal à identifier ses difficultés, ses besoins et ses attentes, à les différencier de ceux de ses collègues, aura du mal à se distinguer de son environnement et en conséquence à confronter ses connaissances avec les sources 
des connaissances nouvelles. Ce savoir sur le savoir - conditionnant le développement cognitif de l'étudiant - ne va pas de soi, comme le remarque M. Perraudeau: «Tous les enseignants savent la difficulté qu'ont les élèves à prendre de la distance sur les façons de travaillen» (Perraudeau 1996: 78).

Dans le cadre de notre pédagogie de l'argumentation écrite, le problème des étudiants consiste à construire une représentation cognitive du texte lu et à se référer à ses représentations des types de textes pour produire des textes argumentés cohésifs-cohérents. L'enseignant pourra donc leur proposer deux types d'activités métacognitives:

- le premier portera sur leurs représentations de la langue et de leur rôle dans le processus d'apprentissage ainsi que sur leurs stratégies mentales;

- le deuxième aura pour objectif la prise de conscience des opérations mentales effectuées lors de la lecture.

La prise de conscience des stratégies mentales, des représentations de la langue et du rôle de l'étudiant dans le processus d'apprentissage peut s'effectuer par des tests pronostics et diagnostics (voir aussi 1.2.2) ainsi que par l'auto-observation en cours et en dehors de cours, suivie de l'échange des remarques entre les étudiants et entre les étudiants et l'enseignant, par exemple: sur leurs modalités et leurs techniques de travail préférés, sur leurs attentes à l'égard de l'enseignant, etc.

La prise de conscience des habiletés et des stratégies de lecture peut constituer une étape autonome du cours ou faire partie intégrante des activités de lecture, par exemple un questionnement avant la lecture: comment allez-vous faire?; et l'auto-observation pendant la lecture suivie de l'échange des remarques et du questionnement après la lecture: avez-vous compris? qu'est-ce qui vous a empêché de comprendre? comment avez-vous procédé? comment pourrait-on s'y prendre différemment?

De plus, en proposant aux étudiants des activités métacognitives, l'enseignant ne peut pas oublier qu'il leur manque le langage pour conceptualiser leur processus d'apprentissage. Il faudra, peut-être, penser à le leur fournir mais au fur et à mesure procédant par l'induction (autrement dit, l'enseignant pourrait demander à l'étudiant de décrire, par exemple comment il procède lors de la lecture et lui donner ensuite le nom de l'opération cognitive).

Ainsi, la tâche de l'enseignant consistera à faciliter à l'étudiant la prise de conscience de son propre apprentissage, ce qui lui permettra de porter un regard critique sur l'efficacité de ses stratégies de lecture et débouchera sur le développement de la capacité à comprendre des textes argumentés.

\subsubsection{BILAN}

En conclusion, au lieu de transmettre le savoir, l'enseignant devra guider le sujet étudiant, coordonner le travail en groupe et encourager les interactions, ce qui demande sans aucun doute des connaissances interdisciplinaires. 


\subsection{BILAN}

Tout compte fait, l'approche interactive de l'enseignement/apprentissage de l'argumentation est orientée sur l'étudiant, et la tâche de l'enseignant consiste à lui faciliter la prise de conscience de son apprentissage et à l'encourager à entrer en interaction avec le texte, l'enseignant et les autres étudiants. Ainsi, l'étudiant et l'enseignant doivent devenir partenaires et coopérer. Ceci est particulièrement nécessaire pour le développement de la capacité à porter un regard critique à l'égard du réel.

\section{L'ENSEIGNEMENT DE LA CAPACITÉ A ARGUMENTER: PROGRESSION ET DEMARCHE}

La progression dans l'enseignement de l'argumentation écrite, que nous présentons ci-après, est envisagée pour deux cent quarante heures de cours réparties en deux ans et adaptée aux besoins des étudiants polonais de niveau avancé. Ses étapes se présentent, grosso modo, comme suit:

- Etape I, dite préliminaire, est prévue pour environ dix heures de cours et constitue l'introduction à la problématique des cours;

- Etape II, envisagée pour une cinquantaine d'heures de cours, contient des activités de la compréhension/production des séquences descriptives et des textes argumentés à orientation descriptive ainsi que des activités de repêrage/identification de la description dans les trois autres types de textes argumentés;

- Etape III, planifiée pour une soixantaine d'heures de cours, se compose d'activitếs de compréhension/production de séquences narratives et de textes argumentés à orientation narrative ainsi que d'activités du repérage/identification de la narration dans les trois autres types de textes argumentés.

- Etape IV, prévue pour une trentaine d'heures de cours, contient des activités de compréhension/production de séquences expositives-explicatives et de textes argumentés à orientation expositive-explicative ainsi que des activités de repérage/ identification de l'exposition-explication dans les autres types de textes argumentés;

- Etape V, envisagée pour environ quatre-vingt-dix heures de cours, se compose d'activités de compréhension/production de séquences argumentatives (justificatives, réfutatives et contre-argumentatives) et de textes argumentés à orientation argumentative se caractérisant par les tendances démonstrative, expositive et dialogique, ainsi que d'activités de repérage/identification de l'argumentation dans les autres types de textes.

Compte tenu des difficultés des étudiants lors de la lecture et de l'écriture des textes argumentés, tout type de texte (séquence) sera traité sous l'angle:

- de la spécificité de son organisation interne et des modalités d'attitude du sujet énonçant, ce qui permettra d'activer (développer) la connaissance des procédés de la mise en page, de la mise en texte et de la mise en discours ainsi que de mettre en relief 
le caractère spécifique du type de texte (séquence) étudié par rapport aux autres types de séquences/textes argumentés;

- de son hétérogénéité séquentielle, ce qui contribuera à souligner les ressemblances entre les types de textes.

A chaque étape de la progression l'étudiant sera, donc, sensibilisé aux types d'arrangements textuels (tabulaire/hiérarchique, chrono-logique et logique) et aux modalités d'attitude du sujet énonçant (autrement dit aux rapports entre le sujet énonçant et l'instance d'énonciation et aux modalités d'implication du sujet énonçant dans le savoir énoncé). Il reste à préciser que nous commençons par la description et par la narration car leurs schémas formels et leurs objets sont les plus familiers pour nos étudiants. De plus, nous admettons que l'évaluation et l'auto-évaluation feront partie des cours.

Afin d'encourager - à chaque étape de la progression - des interactions entre l'étudiant et les sources de connaissances nouvelles, nous adoptons la démarche qui s'inspire librement de la lecture interactive (voir 1.2.1). Ce qui constitue les principaux atouts de cette dernière, c'est qu'elle contribue à activer des connaissances de faible niveau et de haut niveau ainsi qu'à faire alterner des modalités de travail et des stratégies mentales utilisées par les étudiants. De plus, elle offre la possibilité de concevoir plus qu'une séquence didactique à partir d'un seul et même texte et débouche sur des actvivités d'écriture.

\subsection{PREMIÈRE ANNÉE D'APPRENTISSAGE}

Puisque la description et la narration sont les types de textes les plus familiers pour les étudiants polonais, nous proposons de les traiter en première année d'apprentissage, en interaction avec l'exposition-explication et l'argumentation. En début d'apprentissage, l'étudiant du niveau avancé sait, plus ou moins, distinguer les quatre types de textes argumentés. Il est capable de caractériser, brièvement, les textes argumentés à orientations descriptive et narrative. Ce qui lui pose problème, ce sont les caractéristiques de deux autres types de textes ainsi que leur compréhension/production. De plus, il ne remarque pas de ressemblances entre les types de textes, ce qui ne lui facilite ni l'engrangement de connaissances nouvelles ni la compréhension/production de textes. Ainsi, avant de proposer des activités orientées sur la compréhension/production de la description et de la narration, il est indispensable d'initier l'étudiant à la problématique des cours ainsi que de connaître ses représentations et ses besoins.

\subsection{1. ÉTAPE PRÉLIMINAIRE}

Planifiée pour les dix premières heures de cours, l'étape préliminaire a pour objectif, comme nous venons de le mentionner, de préciser à l'étudiant la problématique des cours. Avant de lui proposer des activités orientées sur la 
compréhension/production des types de textes particuliers, il est donc important de préciser ses droits et ses devoirs, de même que de prévoir des séances consacrées à la prise de conscience des stratégies de la lecture en langue maternelle/étrangère, des types de textes ainsi que des rapports entre le texte et le contexte d'énonciation.

A la fin de cette étape, l'étudiant doit savoir:

- évaluer l'efficacité de ses habitudes d'apprentissage;

- évaluer l'état de ses connaissances et le confronter avec les attentes de l'enseignant et la finalité des cours;

- dresser une liste de ses besoins;

- s'interroger sur l'organisation de son apprentissage.

2.1.1.1. «CONTRAT D'APPRENTISSAGE DE LARGUMENTATION ÉCRITE»

Le «contrat d'apprentissage de l'argumentation écrite» est une formule métaphorique qui vise à rendre compte des droits et des devoirs de l'étudiant. Pour les préciser, il s'avère indispensable de partir de ses représentations sur l'enseignement/apprentissage d'une langue étrangère (la définition des concepts de langue, d'apprentissage d'une langue étrangère, de connaissance d'une langue étrangère, de rôle de l'enseignant, etc.), sur l'argumentation (la définition du concept d'argumentation, des savoirs et des savoir-faire nécessaires pour argumenter, etc.) et sur lui-même (les grands traits de sa personnalité, sa motivation, son rôle dans le processus d'enseignement/apprentissage, son style cognitif, etc.). Les techniques de travail qui s'y prêtent bien, ce sont, par exemple des questionnaires et des tests que l'étudiant pourraient remplir individuellement en classe ainsi que l'auto-observation de ses habitudes d'apprentissage et une prise de notes.

\subsubsection{PRISE DE CONSCIENCE DES HABILETES ET DES STRATEGIES DE LECTURE EN LANGUE MATERNELLE ET EN LANGUE ÉTRANGÈRE}

La différence entre la lecture en langue maternelle et en langue étrangère réside dans l'application des habiletés et des stratégies. Un lecteur habile en langue maternelle, notre étudiant procède d'habitude par le déchiffrage et par la traduction en langue étrangère. Pour encourager la réflexion sur la compréhension des textes en langue maternelle et en langue étrangère, l'enseignant peut proposer aux étudiants, d'abord de lire un texte en polonais en se chronométrant et de remplir, après la lecture, le test évaluant sa compréhension; et ensuite de procéder de la même manière avec un texte en français en autorisant l'emploi de dictionnaires. Bien entendu, les textes triés pour cette activité doivent appartenir au même type de discours et se caractériser par un schéma formel, un objet et une longueur semblables. Une autre variante de la même activité consistera à demander aux 
étudiants de lire en polonais et en français les quatre types de textes argumentés, ce qui contribuera à souligner le rôle des schémas formels et de l'objet du texte pour sa compréhension/production, et s'enchaînera aux séances consacrées à la prise de conscience des types de textes (voir 2.1.1.3).

De plus, les séances de prise de conscience des habiletés et des stratégies de lecture constituent une bonne occasion pour souligner l'impact des procédés de la mise en page sur la compréhension des textes. Afin de le montrer, on pourrait proposer la lecture d'un court texte (à orientation descriptive, narrative, expositive-explicative ou bien argumentative) rédigé en deux versions: l'une conforme aux règles conventionnelles de la mise en page et l'autre transgressant ces règles. Les étudiants - divisés en deux sous-groupes - devraient lire individuellement en se chronométrant l'une ou l'autre version du même texte.

En somme, la prise de conscience et la confrontation des démarches effectuées lors de la lecture en polonais et en français pourront aider l'étudiant:

- à évaluer le taux de leur efficacité;

- à identifier les sources de ses difficultés à comprendre le texte en langue étrangère;

- à réfléchir sur les démarches qu'il pourrait effectuer pour améliorer la compréhension des textes lus en français;

- à dresser une liste de ses besoins.

De son côté, l'enseignant pourra mieux adapter les activités aux besoins des étudiants.

2.1.1.3. TYPES DE SÉQUENCES ET TYPES DE TEXTES: INTRODUCTION À LA TYPOLOGIE SÉQUENTIELLE

La prise de conscience des types de séquences élémentaires et de leur combinaison au niveau textuel a pour but d'amener l'étudiant à se rendre compte:

- des différences et des ressemblances dans l'organisation interne des types de textes;

- des rapports entre le schéma formel du texte et son objet.

Puisque les étudiants arrivent avec un certain savoir sur les types de textes - en principe ils sont capables de distinguer la description, la narration et l'argumentation - la tâche de l'enseignant consistera à le compléter et à le systématiser. Les activités suggérées, ici, sont les suivantes:

- le travail sur un tri de textes appartenant aux divers types de discours que l'étudiant devra classer en précisant les critères de son classement;

- une variante du test d'appariement qui consistera à demander aux étudiants de relier les types de textes avec les diverses propositions des schémas de contenu. 
Les séances consacrées à la prise de conscience des types de textes et des rapports entre le type de texte et son objet permettront à l'étudiant de systématiser son savoir et de reconnaître ses besoins. De plus, elles contribuent à justifier l'approche interactive et les étapes de la progression.

\subsubsection{MODALITÉS D'ATTITUDE DU SUJET ÉNONÇANT}

Les étudiants se rendent rarement compte que tout texte cherche l'acceptabilité émotionnelle et/ou intellectuelle du lecteur-cible. Ainsi, avant de leur proposer des activités orientées sur la compréhension/production des types de textes, il s'avère nécessaire d'attirer leur attention sur:

- les comportements énonciatifs du sujet communiquant (le locuteur situationnel ${ }^{1} /$ polémique $^{2}$, l'énonciateur textuel $l^{3} /$ intertextuel $^{4}$ );

- les rapports entre le sujet énonçant et le cadre spatio-temporel des faits/savoirs énoncés (il s'agit de distinguer les discours autonomes et impliqués);

- les rapports entre le sujet énonçant et les faits/savoirs énoncés (l'évaluation des rapports sur les axes: vrai/faux/incertain, bon/mauvais, quantité/qualité et affectif).

L'objectif des réflexions sur des modalités d'attitude du sujet énonçant est de donner aux étudiants un aperçu des procédés de la mise en discours indispensables pour l'interprétation de la visée pragmatique du texte - qui seront traités à fond au cours des étapes suivantes de la progression. Ainsi, comme dans le cas précédent, nous proposons un travail sur le tri de textes appartenant aux divers types de discours dont la longueur ne dépassent pas la moitié d'une page du format A4. La tâche des étudiants consistera à lire des textes et à remplir un questionnaire (par exemple à choix multiple) pour:

- évaluer les faits/savoirs énoncés selon les degrés de leur assertion (l'évaluation de type vrai/faux/incertain) et de leur axiologisation (l'évaluation de type bon/mauvais);

- estimer le degré d'engagement émotionnel du sujet énonçant;

- identifier le cadre spatio-temporel des faits/savoirs énoncés;

- situer le sujet énonçant par rapport à l'espace-temps des faits/savoirs énoncés.

En somme, l'essentiel consiste à donner aux étudiants un «avant-goût» de l'ensemble des items qui seront taités lors des étapes de la progression.

\footnotetext{
' Le locuteur situationnel se manifeste explicitement à la surface du texte, ce qui se traduit par exemple par le recours à la première personne.

${ }^{2}$ Dans le cas du locuteur polémique, le texte porte des traces du locuteur et du destinataire (par exemple: Entrez s.v.p.. Donne-moi ce livre!).

${ }^{3}$ L'énonciateur textuel s'efface derrière les tournures impersonnelles, le groupe nominal ou le pronom gênérique on.

${ }^{4}$ L'énonciateur intertextuel peut être convoqué aussi bien par le locuteur situationnel/polémique que par l'énonciateur textuel d'une manière directe (par exemple le recours au discours direct/indirect) ou indirecte (il s'agit des présupposés et des sous-entendus).
} 
En conclusion, l'étape préliminaire a pour finalité de préparer les étudiants aux étapes suivantes, de les encourager à réfléchir sur leur propre apprentissage, leurs besoins et les attentes de l'enseignant. Cette étape permettra à l'enseignant de connaître ses étudiants, c'est-à-dire leur représentations et leur besoins, et d'y adapter, dans la mesure du possible, les techniques et les modalité du travail ainsi que les contenus des cours. De plus, elle constitue une bonne occasion pour introduire des notions clefs, (par exemple énonciateur textuel/intertextuel, locuteur situationnel/polémique, subjectivèmes, schéma formel, séquence, etc).

\subsubsection{DÉCRIRE}

Prévue pour une cinquantaine d'heures de cours, l'étape consacrée à l'enseignement/apprentissage de la description a pour objectif d'amener l'étudiant:

- à comprendre des séquences descriptives et des textes argumentés à orientation descriptive (il s'agit de reconstruire leur schéma formel et d'interpréter les modalités d'attitude du sujet énonçant, c'est-à-dire les modalités de qualification et de distanciation);

- à savoir inférer des liens interphrastiques;

- à produire des séquences descriptives selon quatre variantes principales du schéma formel descriptif (verticale, latérale, en approche versus en recul, temporelle) (Adam \& Petitjean 1989: 82);

- à rédiger des séquences descriptives de type voir, faire, dire (Adam \& Petitjean 1989: 93-94);

- à enchaîner des séquences descriptives à l'aide des syntagmes verbaux composés de verbes de perception, d'action ou de parole;

- à savoir identifier dans le texte argumenté à orientation descriptive d'autres types de séquences;

- à savoir identifier la séquence descriptive dans les autres types de textes argumentés;

- à savoir situer la description par rapport aux autres types de textes argumentés (c'est-à-dire savoir préciser en quoi consiste sa spécificité par rapport aux autres types de textes argumentés et ses ressemblances avec les autres types de textes argumentés).

Nous commençons le travail sur la description par des activités de repérage (à travers des séquences descriptives et des textes argumentés à orientation descriptive) d'un côté des procédés sous-tendant explicitement l'organisation tabulaire/hiérarchique de la description (à savoir des liages anaphoriques/coréférentiels et des organisateurs énumératifs), de l'autre côté des procédés permettant de préciser l'attitude du sujet énonçant (c'est-à-dire des marques de la localisation spatio-temporelles déictiques, co-textuelles et absolues, des temps 
verbaux absolus/co-textuels ainsi que des subjectivèmes modalisateurs, axiologiques, non-axiologiques et affectifs).

Le travail sur le schếma formel de la description et sur l'attitude du sujet énonçant implique, bien entendu, la sensibilisation de l'étudiant à la convocation indirecte de l'énonciateur intertextuel (autrement dit aux contenus et aux liens présupposés et sous-entendus). Afin d'évaluer l'engrangement de nouvelles connaissances, le repérage des procédés en question devra être suivi des activités de production.

Les activités de repérage/application des liages anaphoriques/co-référentiels et des organisateurs énumératifs permettent à l'étudiant d'identifier/appliquer: éclaté);

- les types de progression thématique (â thèmes constant, linẻaire, dérivé et

- quatre variantes principales du schéma formel de la description;

- des marques linguistiques (les verbes de perception, d'action et de parole) assurant l'enchaînement des séquences descriptives et leur insertion (emboîtement) dans les autres types de textes argumentés.

Elles l'amènent, par la suite, à reconnaître: ratifs;

- la fonction des liages anaphoriques/co-référentiels et des organisateurs énumé-

- la spécificité de l'organisation interne de la séquence descriptive et du texte argumenté à orientation descriptive.

En ce qui concerne les activités de repérage/application des référentiels spatio-temporels et de différentes catégories de subjectivèmes, elles conduisent l'étudiant:

- à situer le sujet énonçant (l'énonciateur textuel/intertextuel, le locuteur situationnel/polémique) par rapport à l'espace-temps d'énonciation (il s'agit de savoir distinguer les discours impliqués versus autonomes et évaluer l'effet énonciatif qui ressort de l'emploi des temps verbaux absolus/co-textuels et de différentes catégories des référentiels spatio-temporels);

- à évaluer les rapports entre le sujet énonçant (l'énonciateur textuel/intertextuel, le locuteur situationnel/polémique) et les faits/les savoirs énoncés.

En bref, les activités mentionnées ci-dessus débouchent sur:

- l'identification du type d'acceptabilité (émotionnelle et/ou intellectuelle) visée par la description et l'effet qui en ressort;

- un classement des moyens linguistiques/non-linguistiques contribuant à identifier les modalités de qualification (par exemple des subjectivèmes axiologiques, le recours à l'exclamation) et de distanciation (par exemple des subjectivèmes non-axiologiques);

- un classement des objets de la description (il s'agit des objets rélévant de l'appréhension perceptive du réel) (Adam \& Petitjean 1989: 75-76);

- une mise en relief du champ des valeurs auquel peut se référer la description (par exemple des valeurs relévant de l'esthétique). 
Après une série d'activités orientées sur le repérage/application des procédés décidant de la spécificité de la séquence descriptive, nous proposons des activités d'identification de la description dans les autres types de textes argumentés. Dans un premier temps, la tâche de l'étudiant consiste à identifier (encadrer) des séquences descriptives dans des textes argumentés à orientations narrative, expositive-explicative et argumentative ${ }^{5}$. Dans un deuxième temps, nous lui demandons de caractériser l'organisation interne et la visée pragmatique des séquences encadrées ainsi que d'observer comment elles s'articulent dans les textes particuliers. Ces activités d'identification/observation doivent être, bien entendu, suivies des activités de production orientées sur l'application des procédés linguistiques assurant l'articulation/emboîtement des séquences descriptives dans les autres types de textes argumentés.

En somme, il s'avère important de concevoir des activités d'une part de manière à développer les connaissances des procédés sous-tendant l'organisation interne de la séquence descriptive et des procédés permettant d'évaluer l'attitude du sujet énonçant; d'autre part de façon à les systématiser. A la fin de cette étape l'étudiant doit savoir non seulement rédiger des séquences descriptives et des textes argumentés à orientation descriptive mais aussi situer la description par rapport aux autres types de textes argumentés.

Pour illustrer l'étape consacrée à la description, nous présentons ci-dessous une séquence didactique qui porte sur l'identification de la modalité d'implication du sujet énonçant dans les faits/savoirs énoncés et débouche sur une prise de conscience de l'acceptabilité visée par la description. Elle est conçue à partir de l'article Elle est pourtant belle, «La mutante» (Ouest-France, le 5 septembre 1995).

\section{DD SÉQUENCE DIDACTIQUE (1)}

\section{Elle est pourtant belle, «La Mutante»}

C'est la dernière des victimes de l'épidémie qui pousse les top-models devant la caméra. On n'a pas seulement embauché Natasha Henstridge pour qu'elle montre son joli minois. Dans «La Mutante» (sortie en France le 27 septembre), elle est une horrible créature d'outre espace.

Elle est vive, souriante et radieuse dans un ensemble jaune-soleil qui fait miroiter ses atours. Vous l'avez deviné, Natasha Henstridge est craquante. Une rafraîchissante oassis de féminité dans un festival qui, avec Eastwood et Costner en attendant De Niro et Nicholson, s'est offert un vingt-et-unième millésime haute-de-gamme certes, mais tendance macho.

${ }^{5}$ Puisque les séquences prototypes sont à identifier dans tout type de discours (bien entendu adaptées à ses traits particuliers), l'enseignant peut choisir le discours-support ả son grè. 
On apprécie d'autant plus sa compagnie qu'on la sait à Deauville sans danger. Tous ceux, bellâtres, godelureaux et fanfarons, qui l'ont approchée à Los Angeles quand elle était «La Mutante» ne peuvent pas en dire autant. Dans ce film de Roder Donaldson (Le Bounty, Sens unique) qui marque son entrée en cinéma, la nouvelle coqueluche des magazines de mode est une horrible créature qui dévore ses victimes pour assurer sa croissance. Et elle met les petits plats dans les grands pour se rassasier.

Le cinéma nous a gavê de telles histoires. Il était difficile à celle-ci, malgré l'ambition qu'elle en avait de nous faire oublier Alien. On retrouve les éternels mêmes procédés qui ne peuvent plus provoquer suspens ni effroi.

Mais jamais du moins un monstre n'avait trouvé refuge derrière une silhouette aussi avenante. Il fallait à Natasha Henstridge l'insouciance de ses vingt ans pour démarrer dans la carrière avec une telle prestation: «Maintenant, je suis tranquille, avance-t-elle avec lucidité. Je suis sûre de ne pas être cataloguée dans un type de rôle. On me proposera forcément des choses différentes». Amusée et intriguée, elle a regretté de ne pouvoir préparer sa composition: «J'aurais souhaité discuter avec des extra-terrestres pour me documenter, mais je n'en ai pas trouvé».

C'est en arrivant sur le plateau le premier jour de tournage qu'elle a ressenti ce qui doit transiter dans la tête d'une créature débarquant d'un autre monde. Elle venait de l'univers de la mode, elle s'est retrouvée aux côtés de Ben Kingsley, Alfred Molina et Forest Whitaker: «J'étais très intimidée, je me demandais ce que je faisais là au millieu de gens prestigieux et que je ne connaissais. J'étais vraiment comme un extraterrestre» Elle a trouvé ses marques dans ce nouveau monde. Elle tourne actuellement aux côtés de Christophe Lambert chez qui elle provoque une poussée d'«Adrénaline». «La Mutante» n'a pas abandonné ses maléfices.

Quest-France, le 5 septembre 1995

Pierre FORNEROD

\section{PRÉLECTURE}

* Objectifs: 1) savoir reconnaître la subjectivité de l'évaluation axiologique; 2) prendre conscience de la spécificité de l'appréhension par la voie des sens; 3 ) encourager la réflexion sur l'acceptabilité suscitée par ce mode d'appréhension du réel;

* Support: une dizaine de photographies (numérotées) représentant des femmes d'âge différent, provenant - de préférence - d'époques distinctes et des quatre coins du monde;

* Modalité de travail: individuel, échange des points de vue;

* Durée: environ 15 minutes.

Consignes: Regardez les photographies et notez les numéros de celles qui présentent selon vous des femmes belles. Comparez les résultats entre vous. Serait-il possible de «justifier» votre choix d'une manière objective?

\section{LECTURE-RECHERCHE}

* Objectif: savoir définir la modalité d'implication du sujet énonçant dans des faits/savoirs énoncés; 
* Supports: texte de l'article et grille de lecture;

* Entrée dans le texte: par des comportements énonciatifs du sujet communiquant;

* Modalité de travail: en sous-groupes (par exemple de quatre personnes);

* Durée: environ 30 minutes.

Consignes: Dans le texte de l'article, repérez et soulignez les expressions axiologiques se rapportant au festival, au film ('La mutante') et à Natasha. Tenez compte des comportements énonciatifs du sujet communiquant (locuteur situationnel/polémique, énonciateur textuel/intertextuel) ainsi que de la valeur de l'évaluation axiologique (évaluation appréciative versus évaluation depréciative). Après avoir échangế les résultats entre vous précisez la modalité (les modalités) d'implication du sujet énonçant.

\subsubsection{NARRER}

Comme dans le cas précédent, l'étape consacrée à la compréhension/ production de la narration (planifiée pour une soixantaine d'heures de cours) se compose des activités orientées sur:

- l'identification/application des procédés sous-tendant l'organisation chronologique de la séquence narrative et du texte argumenté à orientation narrative (à savoir des marques de la localisation temporelle, des organisateurs temporels, des temps verbaux, des connecteurs et des expressions à valeur de connecteurs);

- l'inférence des liens manquant (intraphrastiques et interphrastiques);

- l'interprétation des contenus implicites (présupposés et sous-entendus);

- l'identification/application des procédés gérant des rapports entre le sujet énonçant et le cadre spatio-temporel des faits/savoirs énoncés (c'est-à-dire des marques de la localisation spatio-temporelle, des temps verbaux absolus/co-textuels ainsi qu'entre le sujet énonçant et les faits/les savoirs ênoncés (à savoir des subjectivèmes).

Les objectifs de cette étape sont analogues à ceux de l'étape précédente, c'est-àdire que l'étudiant doit être capable:

- de comprendre des séquences narratives et des textes argumentés à orientation narrative, ce qui revient à savoir reconstruire le schéma formel de la narration et interpréter les modalités d'attitude du sujet énonçant (l'attitude de qualification/ distanciation);

- produire des séquences narratives en s'appuyant sur le schéma narratif prototype (voir Adam: 1994: 93-105);

- établir un cadre spatio-temporel pour des faits/savoirs énoncés;

- se servir de différentes catégories de subjectivèmes pour nuancer ses rapports (en tant que sujet communiquant) avec des faits/savoirs énoncés;

- savoir distinguer dans le texte argumenté à orientation narrative les autres types de séquences;

- savoir identifier la séquence narrative dans des textes argumentés à orientations expositive-explicative et argumentative; 
- savoir préciser en quoi consiste la spécificité de la narration par rapport aux autres types de textes argumentés et en quoi elle est semblable aux autres types de textes argumentés.

En ce qui concerne l'organisation des activités, elle est semblable à celle de l'étape précédente. Nous travaillons donc sur des séquences narratives et des textes argumentés à orientation narrative ainsi que sur des textes argumentés à orientations expositive-explicative et argumentative.

En somme, à cette étape-ci l'accent est mis sur:

- le rôle des référentiels spatio-temporels, des organisateurs temporels et des connecteurs logiques dans l'organisation d'informations (en comparaison avec la fonction des liages anaphoriques/co-référentiels et des organisateurs énumératifs);

- le type d'acceptabilitê visée par la séquence narrative (le texte argumenté à orientation narrative);

- le système de valeurs (les systèmes de valeurs) auquel peut se référer la séquence narrative (le type argumenté à orientation narrative).

En même temps, nous continuons le travail sur:

- les rapports entre le sujet ênonçant et l'instance d'énonciation;

- les modalités d'attitude du sujet ếnonçant;

- la convocation indirecte de l'énonciateur intertextuel;

- le classement des moyens linguistiques/non-linguistiques utilisés pour nuancer l'attitude du sujet énonçant à l'égard de l'instance d'énonciation et des faits/savoirs énoncés.

Pour illustrer l'étape consacrée à la narration, nous proposons une séquence didactique orientée sur l'identification/application du schéma narratif. Envisagée à partir du reportage La mort inexpliquée de Georges Vanderberke, le sacristain si tranquille de Notre-Dame-de-la-Treille (Le Monde, le 13 novembre 1996).

\section{Séquence didactique (2)}

\section{La mort inexpliquée de Georges Vanderberke, le sacristain si tranquille de Notre-Dame-de-la-Treille}

\section{Lille}

de notre envoyé spécial

(I)

Georges Vanderberke, sacristain bénévole à la cathédrale Notre-Dame de-la-Treille, à Lille, est mort d'un coup de poignard en plein coeur, samedi 2 novembre, dans sa sacristie (Le Monde du 5 novembre). Georges Vanderberke avait cinquante ans, et les enquêteurs de la brigade criminelle tentent de percer les secrets d'une vie sans histoire. Depuis plus de trente ans, «Brave Georges» vivait avec sa mère au Quesne, un quartier résidentiel de Marcq-en-Baroeul, dans la banlieue de Lille. Un lieu cossu, tranquille. La villa des 
Vanderberke était plus modeste. Les Delaise, une riche famille de la région chez qui $\mathrm{M}^{\mathrm{me}}$ Vanderberke fut cuisinière, leur avaient prêté gratuitement le pavillon, après le décès du père, au début des années 70 .

Entre eux, jamais une brouille, jamais un mot plus haut que l'autre. Georges vivait pour et par sa mère. Il la vénérait, littéralement, l'avait installée sur un piédestal au point de la considérer comme une sainte, à en croire le Père Roger Desreumaux, chancelier de l'évêché et secrétaire du conseil épiscopal: "Un jour, il $m$ 'a même dit que sa mère avait vu la Vierge.»Pendant de longues années, Georges s'occupa de sa mère, jusqu'à sa mort, à quatre-vingt-six ans, en juillet dernier.

\section{(II) AMBITIONS MODESTES}

Il y a longtemps, Georges avait étế routier, puis employé dans une brasserie. Elle rêvait pour son fils d'une carrière religieuse et racontait à ses voisines qu'elle aurait aimé le voir prêtre. Lui était moins ambititeux: il espérait bien pouvoir un jour entrer dans un monastère, pour y être jardinier. A défaut, Georges offrait ses services à la paroisse de Notre-Dame-de-la-Treille. Un bénévolat auquel il consacrait, après le décès de sa mère encore plus de temps.

Dans cette cathêdrale inachevếe, à laquelle il manque toujours une façade digne de ce nom, Georges avait trouvé une nouvelle raison d'exister. Sacristain d'occasion, il remplaçait le titulaire de la charge lorque celui-ci avait un empêchement. Pourtant, il persévérait. Il n'avait que cela et ses animaux. Son chien d'abord, un fidèle bâtard qu'il appelait simplement "mon chien». Un âne et un bouc aussi, dont il s'occupait avec une tendre affection. Lorsqu'il s'est retrouvé seul, sans moyens financiers, il a préféré faire couper son téléphone plutôt que de se séparer de ses animaux.

Georges vivait au jour le jour, se souciant peu de son apparence et de ses besoins propres. Quelques semaines avant sa mort, il avait même refusé - sursaut d'orgueil ou lassitude? - d'aller signer un dossier de demande de RMI que la famille Delaise avait constitué pour lui. Devenir RMiste, quitter sa maison pour un appartement, comme on le lui avait conseillé, c'était trop lui demander.

Depuis l'ouverture d'un chantier de fouilles archéologiques, le parking de la cathédrale Notre-Dame-de-la-Treille est entouré de palissades. Une faune interlope a colonisé ce no man's land à l'abri des regards. Georges faisait part de ses inquiétudes, de son incapacité à faire face à l' «invasion». Régulièrement, il retrouvait des boîtes de bière vides et des seringues ou des préservatifs usagés. «Parfois, il morigénait tout ce petit monde, raconte le Père Desreumaux, il essayait de les faire déguerpir, sans grand succès».

Georges Vanderberke aurait donc été victime d'un crime crapuleux, commis par un mariginal à la recherche d'un peu d'argent: le matin du meurtre, les funérailles du doyen du chapitre avaient eu lieu, et la quête avait apportế une coquette somme, gardée dans le coffre de la sacristie. Aucun vol n'a été constaté, mais quelques semaines plus tôt, les troncs de Notre-Dame-de-la-Treille avaient été visités. Au mois de mai, l'abbé Bernard, soixante et onze ans, avait été blessé à l'abdomen dans le presbytère de l'église Saint-Maurice, par un jeune toxicomane. Plus récemment, une vieille dame avait été frappée au visage dans l'église Saint-Sauveur et s'était fait voler son chéquier par un inconnu qui a réussi à prendre la fuite. 


\section{(III) UN SEAU D'ESSENCE ENFLAMMÉE}

Qui d'autre aurait pu en vouloir à Georges? On ne lui connaissait aucune famille, à part une vague marraine du côté d'Anderlecht, en Belgique. «Penser qu'il aurait pu avoir des ennemis relève de l'affabulation», affirment ses voisins. Certains, pourtant, rappellent que la vie bien rangée de Georges avait ếté quelque peu perturbée ces derniers temps. A la mi-octobre, il avait été réveillé en pleine nuit par une odeur de brûlé. Un ou des inconnus avaient déposé, à l'arrière de sa maison, un seau en plastique rempli d'essence enflammée. L'incendie commençait à attaquer un volet lorque Georges réussit à l'éteindre. Curieusement, cette nuit-là, son chien n'avait pas aboyé. Hormis quelques cambriolages, le quartier du Quesne n'avait jamais connu d'actes de cette nature. Georges, lui en avait été très affecté et s'en était ouvet à son entourage. «Depuis, il ne se sentait plus en sécurité», se rappelle Léon Perlinski, un voisin.

Difficile de faire un lien entre cet incident et le meurtre. La vie de Georges Vanderberke était bien trop lisse, et le Père Desreumaux préfere replacer sa mort «dans une perspective chrétienne». "C'est peut-être un signe d'espérance, confesse-t-il. Il a enfin retrouvé la seule personne qui comptait pour lui: sa mère».

Le Monde, le 13 novembre 1999

Acacio Pereira

\section{PRÉLECTURE}

* Objectifs: 1) entraîner l'étudiant à identifier les composantes du schéma classique de la narration (voir la grille de lecture ci-après), ce qui lui permettra de le confronter au schéma narratif du reportage et par la suite de prendre conscience des possibilités de le transformer; 2) inicier l'étudiant à la lecture du reportage et lui faciliter l'identification du schéma narratif du reportage;

* Supports: 1) contes rédigés à domicile en groupes de $4-5$ personnes; 2) grille de lecture (voir ci-dessous);

* Modalité de travail: en petits groupes dont chacun présente son conte et écoute à son tour la présentation de leur collègue en remplissant la grille;

* Durée: environ 45 minutes.

Consignes : Écoutez les contes racontés par vos collègues et remplissez la grille ci-dessous.

\begin{tabular}{l|lll|l|}
\hline ETAPES & CONTE 1 & CONTE 2 & CONTE 3 & CONTE 4... \\
\hline Équilibre & & \\
Complication & & \\
Action & & \\
Résolution & & \\
Nouvel équilibre & & \\
\hline
\end{tabular}




\section{LECTURE-RECHERCHE}

* Objectif: savoir comparer un schéma narratif particulier avec le schéma prototype de la narration;

* Support: 1) reportage «La nort inexpliquée de Georges Vanderberke, le sacristain si tranquille de Notre-Dame-de-la-Treille; 2) grille des thèmes locaux (voir cidessous);

* Modalité de travail: en sous-groupes par exemple de 5 personnes;

* Entrées envisagées: par thèmes locaux;

* Durée: 30 minutes environ.

Consignes: Dans le texte de l'article, repérez et encadrez les thèmes locaux énumérés dans la grille ci-dessous. Ensuite, remplissez la grille.

\section{Themes locaux}

\section{le meurtre de Georges}

la vie de Georges avant la mort de sa mère

la mort de Mme Vanderberke

le travail à la cathédrale

\section{les intrus autour de la cathédrale}

les incidents précédant le meutre de Georges

Lisez attentivement ces thèmes locaux et évaluez la signification des événements constituant leur objet pour la vie de Georges Vandenberke en attribuant:

$(+)$ pour l'amélioration

$(\sim)$ pour la perturbation

$(=)$ pour l'équilibre

(!) pour la dégradation

$(\sim)$ pour le déséquilibre

Essayez de reconstruire le plan du reportage (faites son schéma)

\section{POSTLECTURE}

* Objectif: tester les capacités à appliquer le schéma formel de la narration et à insérer la séquence descriptive dans la narration.

Consignes: Racontez. une histoire en s'appuyant sur le schéma du reportage et en y insérant une description depréciative (paysage, personne, animal, chose, etc.) 
Pour conclure, à la fin de la première année d'apprentissage, les étudiants auront déjà un aperçu global des procédés - de la mise en page, de la mise en texte et de la mise en discours - indispensables pour la compréhension/production des textes argumentés. Ils seront, de plus capables de situer la description et la narration par rapport aux autres types de textes argumentés. Ci-dessous nous récapitulons leurs savoir-faire particuliers.

\section{Niveau et type de structuration textuelle: Microstructure et Superstructure}

distinguer/appliquer:

- arrangements textuels d'ordre tabulaire/hiérarchique;

- arrangements textuels d'ordre chrono-logique;

- marques de la localisation spatio-temporelle (déictiques/co-textuelle/absolues);

- temps verbaux absolus/co-textuels;

- différentes catégories de subjectivèmes (modalisateurs, axiologiques, non-axiologiques et affectifs);

\section{Niveau et type de structuration textuelle: Macrostructure et Superstructure}

\section{comprendre/produire:}

- séquences descriptives;

- textes argumentés à orientation descriptive;

- séquences narratives;

- textes argumentés à orientation narrative;

$\varpi$ inférer:

- des liens intraphrastriques/interphrastiques;

identifier/interpréter:

- des contenus implicites;

$\checkmark$ identifier:

- Séquences descriptives et narratives dans les autres types de textes; et narrative;

- les autres types de séquences dans les textes argumentés à orientation descriptive

$\checkmark$ préciser: narration;

- types d'acceptabilité (émotionnelle/intellectuelle) visée par la description et par la

- $\quad$ systèmes de valeurs sur lesquels s'appuient les séquences descriptives et narratives particulières (textes argumentés à orientation descriptive et narrative particuliers);

- catégories d'objets de la description et de la narration;

Pour mesurer le degré d'acquisition des savoir-faire énumérés ci-dessus, il est, bien entendu, nécessaire de tester les connaissances et les capacités de l'étudiant. 
Cette évaluation de type inventaire est particulièrement recommandée à la fin de la première année d'apprentissage car elle permettra non seulement de classer les étudiants mais aussi de réajuster la suite de la progression à leur niveau.

\subsection{DEUXIÈME ANNÉE D'APPRENTISSAGE}

Il découle de ce qui précède qu'en deuxième année d'apprentissage, nous proposons à l'étudiant des activités orientées sur la compréhension/production de l'exposition-explication et de l'argumentation. L'accent sera mis d'un côté sur leur spécificité (c'est-à-dire sur la spécificité de leur schéma formel et des modalités d'attitude du sujet énonçant) par rapport à la description et à la narration, de l'autre côté sur le caractère hétérogène de leur structure séquentielle (afin de souligner leur rapport avec les autres types de séquence).

\subsubsection{EXPOSER-EXPLIQUER}

Prévu pour une trentaine d'heures de cours, le travail sur l'exposition-explication constitue une bonne occasion d'une part de revoir des procédés soustendant des arrangements textuels d'ordre tabulaire/hiérarchique (caractéristiques pour la séquence descriptive), d'autre part de s'arrêter sur des procédés gérant des arrangements textuels d'ordre logique (c'est-à-dire des connecteurs et des expressions à valeurs de connecteurs) afin de mettre en relief leurs fonctions dans la séquence expositive-explicative par comparaison avec la séquence narrative. Plus précisément, décisifs pour l'identification de la séquence expositive-explicative, les arrangements d'ordre logique ne jouent que le rôle secondaire dans le cas de la séquence narrative.

Afin de souligner la spécificité de la séquence expositive-explicative par rapport aux autres types de séquences, il s'avère nécessaire d'attacher l'attention de l'étudiant sur l'attitude d'objectivation du sujet énonçant et sur l'effet énonciatif qui en ressort, à savoir sur l'adhésion intellectuelle. Ceci doit le conduire à classer des moyens non-linguistiques et linguistiques décidant de la modalité d'objectivation (tels que des subjectivèmes non-axiologiques, des modalisateurs marquant l'évidence des faits/savoirs énoncés, l'affirmation, des connecteurs logiques, etc.) et à les comparer à ceux qui sont représentatifs pour la modalité de qualification/distanciation (par exemple des subjectivèmes axiologiques/non-axiologiques). De plus, nous demandons à l'étudiant de classer des objets sur lesquels porte généralement l'exposition-explication (des objets abstraits et des idées relèvant de l'appréhension intellectuelle) et de les confronter avec ceux qui sont généralement représentatifs pour la description et la narration (des objets relèvant de l'appréhension perceptive). 
Analogiquement aux étapes précédentes, pour activer des connaissances des étudiants et pour vérifier l'engrangement des connaissances nouvelles, nous proposons (à partir des séquences expositives-explicatives et des textes argumentés à orientation expositive-explicative) des activités de repérage et d'identification qui débouchent sur des activités de production.

En somme, à la fin de cette étape l'étudiant doit savoir activer/configuer ses connaissances non-linguistiques, linguistiques, pragmatiques et socio-culturelles pour:

- identifier/appliquer le schéma formel de l'exposition-explication et le comparer aux schếmas formels des séquences descriptive et narrative;

- définir la modalité d'attitude du sujet énonçant et évaluer l'effet énonciatif qui en ressort (c'est-à-dire l'adhésion intellectuelle);

- comparer l'acceptabilité visée par la séquence expositive-explicative (le texte argumenté à orientation expositive-explicative) avec l'acceptabilité cherchée par les séquences descriptive et narrative (les textes argumentés à orientations descriptive et narrative);

- comparer les objets sur lesquels porte d'habitude la description et la narration avec ceux qui sont représentatifs pour l'exposition-explication;

- définir le caractère des prémisses à partir desquelles se développe la séquence expositive-explicative;

- préciser la spécificité de la séquence expositive-explicative par rapport aux autres types de séquences;

- reconnaître des rapports entre la séquence expositive-explicative et les autres types de séquences;

- rédiger une séquence expositive-explicative en s'appuyant sur le schéma formel prototype (le schéma formel prototype de l'exposition-explication comporte le plus souvent une phase de questionnement, une phase de résolution et une phase conclusive).

Pour illustrer l'étape conšacrée à la compréhension/production de l'exposition-explication, nous présentons ci-après une séquences didactique orientée sur l'identification de l'attitude du sujet énonçant et sur l'évaluation de l'effet énonciatif qui en découle.

\section{Déquence didactique (3)}

\section{Les biologistes préparent de nouvelles armes contre l'ostéoporose}

Une femme sur quatre, dans la population blanche, est atteinte par cette maladie du troisième âge, qui affecte aussi les homme. Des équipes scientifiques tentent d'expliquer la relation entre le déficit d'oestrogènes dans le sang et la fragilité des os.

On dénombre chaque année dans le monde 1,7 million de fractures du col du fémur dues à l'ostéoporose, une maladie des os. (...) Dans la population blanche, l'ostéoporose 
touche une femme sur quatre à la ménopause. Mais elle est commune à tous les groupes ethniques et affecte aussi les hommes.

Comme la maladie d'Alzheimer, l'ostéoporose est une maladie du troisième âge. La population vieillissant, notamment dans les pays développés, les scientifiques s'intéressent de plus en plus à cette pathologie, à ses causes, à ses conséquences et aux remèdes possibles. (...)

Pour comprendre l'ostéoporose il faut connaître l'os. (...) Pour faire simple, l'os se compose de deux types de matériaux fondamentalement différents. Les protéines d'abord, comme le collagène; celle de l'os sont solides, résistantes et flexibles. Les minéraux ensuite, de l'hydroxyapathie une variante du phosphate de calcium; ils donnent à l'os sa rigidité. Protéines et minéraux se complètent de façon idéale, mais sont quasiment inutiles l'un sans l'autre. On peut tordre et nouer un fẻmur humain exempt de minéraux. Mais un os sans protếines se brisera au premier pas.

L'os, donc, est issu d'une succession de processus qui le détruisent et le reconstruisent en permanence. Chaque jour, (...) les ostéoclastes détruisent une certaine quantité d'os et (...) les ostéoblastes reconstruisent la substance osseuse, mais sans combler totalement les cavités creusées par les ostéoclastes.

Des centaines, voire des miliers de processus biochimiques influencent la résorption et la formation osseuses dans le cadre de ce remodelage permanent. Ainsi les oestrogènes hormones sexuelles «féminines» - exercent-ils une action importante sur le bilan osseux. Et, si l'ostéoporose touche tout particulièrement les femmes entre deux âges ou déjà âgées, c'est que la concentration d'œstrogènes dans le sang s'effectue après la ménopause, entraînant donc une plus grande fragilité des os. Mais pourquoi diable une hormone sexuelle est-elle ainsi partie prenante dans le bon état des os? Les chercheurs se sont donné pour objectif de lever ce mystère (...) afin d'apprendre à contrôler ces mécanismes dans un but thérapeutique.

On sait, par exemple, que la vitamine D - présente notamment dans l'huile de foie de poisson (...) - est essentielle à la croissance des os. Cette vitamine recueille le calcium des aliments et assure son acheminement vers les ostéoblastes, cellules bâtisseuses de la substance osseuse. Les personnes manquant de vitamine $D$ au cours de leur croissance souffrent de rachitisme, la carence en minéraux ayant pour conséquences des déformations du squelette. Le mécanisme de l'ostéoporose est légèrement différent. Cette affection survient quand la résorption des minéraux l'emporte sur le renouvellement osseux. Il en résulte une porosité accrue des os et un affaiblissement de leurs capacités mécaniques, a l'origine des fractures.

Pour exercer son action, la vitamine doit s'associer à des récepteurs spécifiques à la surface des cellules. Ces récepteurs sont des protéines dont la forme précise est codée par les gènes. Partant du principe que de légères différences génétiques devraient suffire pour que les récepteurs se lient de façon plus ou moins efficace à la vitamine $D$, des chercheurs australiens ont tentê de mettre en évidence une correspondance entre les variations du gène récepteur de la vitamine $\mathrm{D}$ et celle de la densité des minéraux dans les os. Cette équipe, dirigée par les professeurs Elsman et Morisson (Sydney), a publié, en 1994, une étude épidémiologique tendant à montrer que les personnes porteuses d'une certaine forme (ou «allèle») d'un certain gène étaient plus à même de développer l'ostéoporose avec l'âge que celles qui possèdent une autre forme de ce gène.

Selon leurs travaux, le «seuil de fracture» (densitế osseuse à partir de laquelle la fracture est probable) du bas de la colonne vertébrale se situait aux alentours de soixante-six 
ans chez les femmes qui possèdent deux allèles de type $B$ pour le récepteur de la vitamine $D$, alors que les porteuses de deux allèles de type B n'atteignent ce seuil que vers soixante-seize ans. L'influence de ce gène semblait aussi importantes que celle des astrogènes! Le fait qu'une variante naturelle aussi infirme puisse accorder - ou refuser - à une femme dix ans de vie sans fracture a stupéfié les milieux scientifiques. La résorption et la croissance osseuses sont, en effet des phénomènes très complexes contrôlés par une multitude d'hormones et de facteurs de croissance. Des centaines de gènes sont concernés et il semblait étonnant qu'un seul d'entre eux puisse être prédominant à ce point.

Aux États-Unis, en Europe et au Japon, de nombreuses équipes ont donc multipliê les études épidémiologiques pour tenter de retrouver sur des populations différentes ce que leurs confrères australiens avaient détecté sur un échantillon restreint de Blancs australiens d'ascendance anglo-irlandaise. «La plupart de ces études ont été négatives, et certains commencent à émettre de sérieux doutes sur la validité des résultats de l'équipe d'Elsman et de Morisson», affirme le Français Pierre-Jean Meunier (...). Contestés ou pas, ces travaux démontrent qu'une rechereche plus poussée sera nécessaire avant que les rapports précis entre le récepteur de vitamine $\mathrm{D}$ et la densité osseuse ne soient établis avec certitude.

Une étude américaine plus récente vient renforcer encore cette impression. Elle a été mené par Gerard Karsenty et son équipe de l'université du Texas, à Houston, qui ont élevé une lignée de souris incapables de fabriquer leur propre ostéocalcine, l'une de nombreuses protéines liées à la croissance et à la résorption osseuses.

L'ostéocalcine freine la formation osseuese alors que, curieusement, elle est produite par les cellules ostéoblastes qui élaborent cette dernière! (...) Les souris vivant sans ostéocalcine grandissent et se comportent normalement. Elles paraissent même se porter plutôt bien. En l'absence de cette protéine, leur os deviennent plus grands et plus forts, et cela sans troubles manifestes. A quoi donc l'ostéocalcine peut-elle donc servir? Ce sont les œstrogènes qui font la différence. Les souris sans ostéocalcine auxquelles on a retiré les ovaires et qui, de ce fait, souffrent aussi d'une carence en œstrogènes, sont deux fois plus sensibles à l'ostéoporose.

Ce résultat laisse penser que les femmes ménopausées pourraient tirer profit d'un médicament bloquant l'action de l'ostéocalcine, à condition cependant qu'il soit acompagné d'un supplément d'œstrogènes dans le cas d'un traitement hormonal complet de substitution.

Le Monde, le 13 novembre 1996

Henry Gee

* Objectif: 1) savoir définir la modalité d'implication du sujet énonçant dans les faits/savoirs énoncés; 2) savoir évaluer l'acceptabilité visée par le texte argumenté à orientation expositive-explicative;

* Support: article de presse «Les biologistes préparent de nouvelles armes contre l'ostéoporose» (Le Monde, le 13 novembre 1996);

* Modalité de travail: en tandem

* Entrée dans le texte: par thèmes locaux;

* Durée: 30 minutes environ. 


\section{PRÉLECTURE}

Puisque l'article en question se caractérise par une terminologie de spécialité qui pourrait poser des problèmes aux étudiants, nous proposons de leur demander de chercher, à domicile, des informations sur l'ostéoporose en français ou bien en polonais.

\section{LECTURE-RECHERCHE}

Consignes: Dans le texte de l'article, repérez et encadrez les thèmes indiqués ci-dessous

1) Composants de l'os

2) Processus de la destruction et de la reconstruction de la substance osseuse

3) Estrogènes

4) Vitamine D

5) Gène recepteur de la vitamine $D$

6) Ostéocalcine

Consignes: Lisez attentivement les passages encadrés et soulignez de couleurs différentes les subjectivèmes:

axiologiques (bon/mauvais), non-axiologiques (quantité/qualité), modalisateurs (évidents/douteux).

\section{POSTLECTURE}

* Objectif: vérification de l'engrangement des connaissances nouvelles;

* Mode de travail: les étudiants cherchent à répondre aux questions (voir ci-dessous) en sous-groupes;

\section{Questions:}

- Pourquoi le sujet énonçant évite-t-il l'emploi des expressions axiologiques?

- Recourt-il souvent au conditionnel ou au subjonctif?

- Quel temps grammatical et quel mode dominent dans le texte de l'article?

- Comment s'enchaînent les paragraphes (les séquences) de l'article en question?

- Quel type d'adhésion produit cet article, émotionnelle ou intellectuelle?

\subsubsection{ARGUMENTER}

La dernière étape de la progression (environ quatre-vingt-dix heures de cours) est consacrée à l'exploitation des séquences argumentatives et des textes argumentés à orientation argumentative. Son objectif consiste à développer chez l'étudiant les capacités:

- à identifier/appliquer le schéma argumentatif (justificatif, réfutatif et contre-argumentatif) et l'attitude de prise de position; 
- à distinguer les trois variantes du schéma argumentatif dialogique (c'est-à-dire les schémas antithétique $^{6}$, dialectique ${ }^{7}$ et dialogique ${ }^{8}$ ) (Mirabail 1994: 180-181);

- à identifier dans le texte argumenté à orientation argumentative des séquences descriptive, narrative et expositive-explicative;

- à identifier dans le texte argumenté à orientation argumentative les démarches expositive-explicative, démonstrative, «diluée» et dialogique (Boissinot 1992: 41-42);

- à situer la séquence argumentative par rapport aux autres types de séquences;

- à définir le caractère des prémisses à partir desquelles se développe la séquence argumentative;

- à préciser le(s) système(s) de valeurs auquel se réfere l'argumentation;

- à identifier l'objet de l'argumentation.

Pour y aboutir, nous envisageons des activités orientées d'une part sur la distinction/application des constituants du schéma argumentatif (c'est-à-dire: introduction, thèse(s), arguments, conclusion) ainsi que sur les caractéristiques de leurs fonctions dans le texte argumenté à orientation argumentative; d'autre part sur l'identification/application de l'attitude de prise de position.

En ce qui concerne la distinction/application des constituants du schéma argumentatif, il s'avère important d'attirer l'attention de l'étudiant sur les composantes de l'introduction (la présentation du sujet, l'exposé de la problématique, la suggestion du plan) et de la conclusion (la synthèse des arguments et l'élargissement de la pensée ainsi que sur le caractère polémique de la thèse et des arguments, ce qui lui sera d'ailleurs utile pour comprendre/rédiger des textes argumentés à orientation argumentative (y compris la dissertation).

Quant à l'attitude de prise de position, l'essentiel consiste à sensibiliser l'étudiant à la différence entre les arguments affirmatifs dont l'objectif est d'éclairer/illustrer le point de vue énoncé et quasi-logiques qui servent à valider la thèse (Mirabail 1994: 120-121) ainsi qu'à mettre en relief l'effet énonciatif ressortant de leur emploi (c'est-à-dire l'adhésion émotionnelle dans le cas des arguments affirmatifs et de l'adhésion intellectuelle dans le cas des arguments quasi-logiques). Ceci implique d'un côté des activités portant sur:

- le repérage de différentes catégories de subjectivèmes qui conduira l'étudiant à préciser la modalité d'implication du sujet énonçant dans les faits/savoirs énoncés, à définir les systèmes de valeurs auxquels il se réfere pour susciter l'adhésion

${ }^{6}$ Le schẻma antithétique est composé de deux séquences justificatives: l'une pour la thèse A qui se trouve dévalorisée et l'autre pour la thèse $\mathrm{B}$ qui est mise en valeur et validée.

${ }^{7}$ Le schèma dialectique se compose de trois séquences dont la première sert à justifier la thèse $\mathrm{A}$, la seconde a pour objectif de réfuter cette même thèse et la troisième a le caractère contre-argumentatif: elle développe un nouveau point de vue qui dépasse les contradictions soulevées précédemment.

${ }^{8}$ Le schéma dialogique se caracterise par une confrontation d'une thèse $A$ et d'une thèse $B$ dans l'introduction et une confrontation permanente des arguments pour la these $\mathrm{A}$ et pour la thèse $\mathrm{B}$ dans le développement - orientée sur la valorisation de la thèse $\mathrm{B}$. 
émotionnelle du public-cible et à réfléchir sur le caractère des prémissses â partir desquelles se développe l'argumentation;

- le prélèvement des citations qui amènera l'étudiant à identifier l'argument d'autorité - relèvant des arguments affirmatifs - et à reconnaître sa force illocutoire;

- l'identification des séquences descriptive, narrative et expositive-explicative dans le texte argumenté à orientation argumentative, ce qui lui facilitera le repérage de l'exemple et de l'illustration faisant partie des arguments affirmatifs.

De l'autre côté, la sensibilisation de l'étudiant à la différence entre les arguments affirmatifs et quasi-logiques implique des activitếs orientées sur le repérage/classement des connecteurs et des expressions à valeur de connecteurs, compte tenu de leurs fonctions textuelles/discursives (co-orientation et antiorientation des arguments). Ceci entraîne, de plus le travail sur l'identification/interprétation des présupposés véhiculés par les connecteurs prédicats à trois places anti-orientant des arguments (mais, bien que, cependant, pourtant, etc.). Puisque l'étudiant doit déjà savoir identifier/appliquer des arrangements d'ordre logique il s'agit, à cette étape-ci, de systématiser/compléter ses connaissances.

Les activités de repérage et de prélèvement mentionnées ci-dessus seront suivies, comme dans les cas des étapes précédentes, des activités de sémi-production (par exemple la formulation des thèses opposées à partir d'un mot, la mise en ordre des séquences argumentatives de manière à reconstruire la variante antithétique du schéma argumentatif, etc.) et de production des séquences argumentatives (justificative, réfutative et contre-argumentative).

En somme, la présente étape permettra d'une part de revoir et de systématiser des connaissances de l'étudiant, d'autre part de souligner la spécificité de la séquence argumentative et d'attirer l'attention de l'étudiant sur les variantes antithétique, dialogique et dialectique du schéma argumentatif caractéristiques en particulier pour la dissertation. Afin d'illustrer cette étape nous présentons ci-après une séquence didactique dont l'objectif consiste:

- à sensibiliser l'étudiant au rôle des systèmes de valeurs dans l'argumentation ainsi qu'à leur caractère stéréotypé et culturel;

- à l'entraîner à inférer des thèses adverses à partir des suites d'arguments.

\section{Séquence didactique (4)}

\section{Le mythe de la fin du travail par Philippe d'Iribarne}

La résistance persistante du chômage aux multiples thérapeutiques que les gouvernements de tous les bords mettent en œuvre, année après année, conduit assez logiquement à rechercher les moyens de rendre supportables une situation où nombre de ceux qui désirent travailler sont durablement privés d'emploi. On fait remarquer ici ou là que, après tout, le fait de travailler n'a pas toujours été, et de loin, un attribut majeur de 
l'individu bien intégrế dans sa société, que les Grecs ont valorisế une vie de loisirs, qu'au début du siècle l'état de rentier était encore considéré comme parfaitement respectable. Pourquoi, dans ces conditions considérerait-on comme inéluctable que de nos jours l'individu sans travail cesse d'être regardé comme citoyen honorable? Pouvons-nous réellement compter sur pareille évolution du regard social?

On ne peut répondre sérieusement à la question sans distinguer deux formes bien différentes de vie sans travail. La vie de celui qui faisant partie des forts, a dans sa dépendance suffisamment d'esclaves, ou suffisamment de biens, pour en forcer d'autres à travailler pour lui et la vie de celui qui, faible, dépourvu de capacité à assurer sa propre subsistance, en est réduit à dépendre d'autrui pour survivre. C'est bien sûr la vie du premier que les Grecs louaient, pendant qu'ils méprisaient le second. Et quand les sociétés européennes regardaient le riche rentier avec considération, elles traitaient au mieux avec une commisération condescente celui qui mendiait son pain. Comment donc peut être vu, en donnant sens à sa situation, celui que certains de nos contemporains invitent à vivre autrement qu'en travaillant?

Une première lecture se dessine sans doute dans un registre politique et juridique. Chacun, en tant que membre d'une société de citoyens, a droit à la solidarité de ses égaux. Quand la collectivité lui donne les moyens de subsister, elle ne fait que respecter ses droits. A recevoir une aide, on ne ressemble ni aux dominants qui en obligent d'autres à travailler pour eux, ni aux dominés qui dépendent de la bienveillance d'autrui. On reste citoyen, égal en dignité à tous les citoyen. Pourtant, dès qu'on quitte ce registre des principes et des lois pour se situer dans le registre des rapports sociaux quotidiens, cette vision d'égalité civique a du mal à résister aux deux visions traditionnelles de celui qui n'assure pas sa propre subsistance.

Bien sûr, l'image du faible qui quémande et se soumet aux exigences des questionnaires des diverses formes d'aide, à leurs questions inquisitrices, à leurs ingérences dans sa vie la plus intime (ne peuvent-ils pas, s'il se conduit mal, aller jusqu'à lui enlever ses enfants?), et est ainsi réduit à une situation indigne, n'a pas disparu. Combien d'allocataires du RMI vivent leur situation en la regardant à travers cette image? Mais l'autre image, celle du prédateur qui n'attend pas qu'on lui donne mais qui se sert, qui force d'autres à nourrir son oisiveté, n'a pas été oubliée non plus. C'est elle qui anime les réactions populaires face au mauvais pauvre, qui est accusé d'abuser cyniquement de l'aide publique ou qui, mêlant hommes et chiens dans des bandes agressives, fait peur aux honnêtes gens.

Entre ces deux images, de celui qui reçoit et dépend, et de celui qui prend et exploite, il n'est guère de place, parmi les bons citoyens, pour qui ne travaillent pas. Certes, on peut, face à ces réactions du corps social, mettre en avant la capacité imprescriptible des humains à se libérer des pesenteurs du passé, à faire table rase des conceptions anciennes pour construire une société nouvelle. Mais l'expérience de ces derniers siècles montre les limites d'un tel volontarisme. Et, plus encore, n'est-ce pas la vision même d'une société de citoyens rassemblant des individus émarcipés de toute dépendance qui nous fait juger nos congénères à l'aune de l'idéal de celui qui ni ne dépend ni n'a réduit autrui à dépendre?

La fin du travail? Peut-être un jour, mais dans une société fondée sur des principes tellement différents de ceux auxquels nous adhérons qu'elle n'est ni pour aoujourd'hui ni pour demain ni, à coup sûr, pour le siècle à venir.

Le Monde, le 13 novembre 1996 
* Objectifs: 1) savoir inférer des thèses adverses à partir de séquences d'arguments; 2) savoir définir les systèmes de valeurs à partir desquels se développe l'argumentation dans l'article Le mythe de la fin du travail (Le Monde, le 13 novembre 1996);

* Support: texte de l'article (voir plus loin)

* Mode de travail: en tandem;

* Durée: 30 minutes environ.

\section{PRÉLECTURE}

Pour activer et orienter des connaissances des étudiants (il s'agit avant tout des connaissances socio-culturelles), nous leur proposons de noter individuellement toutes les associations avec le mot chômeur et de formuler le stéréotype du chômeur qui fonctionne dans la société polonaise.

\section{LECTURE-RECHERCHE}

Entrées dans le texte: (a) caractéristiques de la situation du chômeur sur le plan civique; (b) caractéristiques de la situation du chômeur sur le plan des rapports sociaux

Consignes pour l'entrée (a): Identifiez les paragraphes qui caractérisent la situation du chômeur sur le plan civique et encadrez-les. Soulignez d'un trait les subjectivèmes axiologiques à valeur appréciative et de deux traits ceux à valeur depréciative.

Consignes pour l'entrée (b): Dans le fragment de l'article, identifiez les paragraphes qui traitent de l'image du chômeur sur le plan des rapports sociaux. Encadrez-les. Soulignez d'un trait les subjectivèmes axiologiques à valeur appréciative et de deux traits ceux à valeur depréciative.

\section{POSTLECTURE}

Consignes: Répondez aux questions ci-dessous

\section{Questions:}

- Comment est évalué le chômeur dans le registre civique et dans le régistre des rapports sociaux?

- Sur quelles valeurs s'appuie le sujet énonçant pour développer son argumentation?

- Avec quelle opinion polémique le sujet énonçant et quel point de vue défend-il?

- Enchaînez ces deux opinions adverses de manière à valoriser le point de vue défendu par le sujet énonçant.

- Comparez le stéréotype de chômeur fonctionnant dans la société française avec son équivalent en Pologne. Y-a-t-il des ressemblances? 
En somme, à la fin de la deuxième année d'apprentissage, l'étudiant sera capable de comprendre et de comparer les quatre types de séquences et les quatre types de textes argumentés (c'est-à-dire leurs schémas formels et leurs visées pragmatiques). Ceci devra améliorer la cohésion et la cohérence de ses écrits ainsi que faciliter indirectement la compréhension/production de la dissertation.

\subsection{BILAN}

La progression, dont nous venons de caractériser les étapes, vise à sensibiliser l'étudiant d'une part aux traits particuliers des types de séquences, d'autre part aux ressemblances entre les types de textes argumentés afin de le conduire à construire des représentations cognitives des textes argumentés. Les activités types - conçues à partir des textes de presse - ne prétendent bien évidemment pas, à l'exhaustivité.

\section{CONCLUSION}

L'approche interactive, dont nous avons caractérisé les principes et les contenus, insiste d'une part sur le développement des habiletés générales de l'individu, (telles que l'esprit critique ou la gestion de son apprentissage), d'autre part sur le développement de sa capacité à comprendre des textes argumentés (autrement dit à construire des représentations cognitives des textes). De plus, son caractère interactif se traduit sur le plan de l'organisation de la progression par l'interaction entre les types de textes (leurs schémas formels et les modalités d'attitude du sujet énonçant) et sur le plan de la démarche par l'interaction entre la lecture et l'écriture (des activités de lecture débouchent sur des activités d'écriture).

Se situant entre l'approche globalisante des phénomènes argumentatifs et l'approche classique de l'écrit identifiant l'argumentation au type de texte, cette approche cherche à répondre aux besoins des étudiants polonais qui ont souvent du mal à situer l'argumentation par rapport aux autres types de textes et à reconnaître des phénomènes argumentifs dans la description, la narration et l'exposition-explication.

En même temps, il est nécessaire de souligner que la présente proposition ne prétend pas à l'exhaustivité. Certains aspects - tels que la polyphonie, l'implicite ou le rôle des valeurs dans l'argumentation - demanderaient sûrement une étude à part. De plus, il serait intéressant de proposer un enseignement comparatif des phénomènes argumentatifs afin de mettre en relief les ressemblances et les différences culturelles entre le discours argumentatif polonais et français. Ceci 
permettrait, entre autres, de mieux comprendre les sources des difficultés des étudiants polonais. En bref, étant un phénomène complexe et hétérogène, l'argumentation offre un répertoire de possibilités d'études inépuisable et nous n'en avons indiqué que quelques-unes.

\section{BIBLIOGRPHIE}

A d a m, J.-M. (1990), Éléments de la linguistique textuelle, Bruxelles: Mardaga.

A d a m, J.-M. (1991), Langue et littérature, Paris: Hachette.

A d a m, J.-M. (1994), Le texte narratif, Paris: Ed. Nathan.

A d a m, J.-M. \& P e t i t j e a n, A. (1989), Le texte descriptif, Paris: Ed. Nathan.

A n s c o m b r c, J.-C. \& D u c r o t, O. (1983), Argumentation dans la langue, Bruxelles: Mardaga.

A n s c o m bre, J.-C., (1991), Dynamique du sens et scalarité, Colloque de Cerisy L'argumentation:

123-145. Textes èditês par A. Lempereur, Liège: Mardaga.

A r y s to te le s, Retoryka-Poetyka. 1988, Warszawa: PWN.

B a i n, D. (1987), D'une typologie à une didactique du texte, Enjeux 11:49-68.

B a u t i e r, R. (1994), De la rhétorique à la communication, Grenoble: PU.

B Iachowska, M. \& Karpińska-Szaj K. (1995), Argumentacja w nauczaniu języka pisanego na filologii romańskiej, Neofilolog 10: 32-40.

B I a c how s k a, M. (1995), Indices argumentatifs dans l'enseignementapprentissage des stratégies d'argumentation au niveau avancé: suggestions didactiques, Neofilolog 11: 10-21.

B l a chow s ka, M. (1999), La cognition et l'enseignement/apprentissage de l'argumentation au niveau avancể de FLE, La pensêe et la langue: 152-160. Kraków: Wydawnictwo Naukowe AP.

B I a i r, Anthony, J., Qu'est-ce que la logique non formelle, Colloque de Cerisy L'argumentation: 79-

89. Textes êdités par A. Lempereur. Liêge: Mardaga.

B o is s i n o t, A. (1992), Les textes argumentatifs, Toulouse: Bertrand-Lacoste.

B o u c h a r e n c, M. \& F e Il e r, M. (1991), Littérature et types de textes, t. 2, Paris: Techniplus.

B r a u d, M. (1991), L'expression écrite et la composition française, Kraków: C \& D International.

Bronckart, J.-P. (1985), Le fonctionnement des discours, un modele psychologique et une méthode d'analyse, Paris: Delachaux \& Niestlé.

C a 11 i a bets ou-Corac as, P. (1982), Situations d'écriture scolaire, Le Français dans le monde $n^{\circ} 167: 28-33$.

Carrell, P. (1990), Rôle des schémas de contenu et des schêmas formels, Le français dans le monde: $n$. spécial, Recherches et application: 16-29.

C i c u r e 1, F. (1982), Conversations écrites, Le Français dans le monde $\mathrm{n}^{0}$ 167: 20-27.

C i cure l, F. (1988), Cohérence textuelle et acquisition d'une compétence de lecture, La Lecture. Cahier $n^{0}$ 6: 189-203.

C i c u r e l, F. (1991), Lectures interactives en langue êtrangère, Paris: Hachette F.L.E.

C icure l, F. (1991), Comprèhension des textes: une démarche interactive, Le Français dans le monde $\mathrm{n}^{0} 243: 40-46$.

Ch ar a u d e a u, P. (1983), Langage et discours, Paris: Hachette.

Ch a r oll e s, M. (1994), Cohésion, cohérence et pertinence du discours, R.I.L.F, $\mathrm{n}^{\circ} 29: 125-151$.

Chat ma n, S. (1991), Arguments et narrations, Colloque de Cerisy L'argumentation: 147-152.

Textes édités par A. Lempereur, Liège: Mardaga.

C orn a ir e, C. (1991), Le point sur... La lecture en didactique des langues, Anjou (Quebec): Centre Educatif et Culturel. 
C o s te, D. (1974), Lire le sens, Le Français dans le monde $\mathrm{n}^{0} 109: 40-44$.

C o t e n t i n- R e y, G. (1992), Le résumé, Paris: Clẻ International.

$\mathrm{D}$ a b è $\mathrm{n}$ e, M. 1974. L'écrit en question, Le Français dans le monde $\mathrm{n}^{\circ}$ 109: 6-9.

De c l ercq. G. (1993), L'art d'argumenter: structures rhétoriques et littéraires, Paris: Ed. universitaires.

D e mo u g i n, F. (1996), Comprendre un ênoncé argumentatif, Travaux de didactique du FLE $\mathrm{n}^{\circ} 35$ : 75-94, CFP.

Descombres-Dé nevaud, M. \& Jespersen, J. Discours rapporté et anaphore conceptuelle dans le discours argumentatif, Actes du VIII ${ }^{\mathrm{e}}$ Colloque Internationnal: Acquisition et enseignement/apprentissage des langues, perspectives et recherches: 487-497.

D u c r o t, O. (1980), Les échelles argumentatives, Paris: Les Ed. de Minuit.

D u c r o t, O. (1984), Le dire et le dit, Paris: Ed. de Minuit.

D u c r o t, O. (1988), Topoï et formes topiques, Bulletin d'études de linguistiques française: 1-14.

$\mathrm{E} \mathrm{h} \mathrm{r}] \mathrm{i}$ c h, M.-F. (1994), Mémoire et compréhension du langage, Lille: P.U.

$\mathrm{G}$ a on a c'h, D. (1987), Théories d'apprentissage et d'acquisition d'une langue étrangère, Paris: Crẻdif/Hatier Collection L.A.L.

$\mathrm{G}$ a u th e r o t, J-M., (1974), Ecrit et apprentissage, Le Français dans le monde $\mathrm{n}^{\circ} 109: 26-31$.

$\mathrm{G}$ u i c h a r d, S. (1990), La dissertation et le commentaire composé, Paris: Ed. Nathan.

Hé n a u t, A. \& Margeri e, Ch. de, (1974), L'écrit, spécificité et diversité, Le Français dans le monde $n^{0} 109: 10-16$.

Hongré, B., Eterste in, C., J o ye ux, M., Le sot, A. (1996), Le texte argumentatif au bac, Paris: Hatier.

Kerbrat-Orecchioni, K. (1980), L'énonciation de la subjectivité dans le langage, Paris: A. Colin.

Kerbrat - Orecchion i, K. (1986), L'implicite, Paris: A. Colin.

K i n c h, W. \& Van D i jk, T. A. (1975), Comment on se rappelle et on résume des histoires, Langages $\mathrm{n}^{0} 40: 98-116$.

$\mathrm{Lu} \mathrm{nd} \mathrm{qu} \mathrm{is} \mathrm{t,} \mathrm{L.} \mathrm{(1993),} \mathrm{La} \mathrm{coherence} \mathrm{textuelle} \mathrm{argumentative:} \mathrm{illocution,} \mathrm{intention} \mathrm{et} \mathrm{engagement}$ de consistance, Revue québécoise de linguistique, vol. $22, \mathrm{n}^{0} 2$ : 109-138.

$\mathrm{M}$ a in g u e n e a u. D. (1987), Nouvelles tendances en analyse du discours, Paris: Hachette.

M i r a b a i l, H. (1994), Argumenter au lycée, Bertrand-Lacoste, CRDP Midi-Pyrénées.

M o e s c h l e r, J. (1985), Argumentation et conversation, eléments pour une analyse pragmatique du discours, Paris: Hatier.

M o e s c h l e r, J. (1994), Structure et interprétabilité des textes argumentatifs, Pratiques n 84: 93-III.

M o e s h le r, J. \& R e boul A. (1994), Dictionnaire encyclopédique de pragmatique, Paris: Ed. du Seuil.

Mo ir a nd, S. (1975), Le rôle anaphorique de la nominalisation, Langue Française $n^{\circ} 28: 60-78$ Paris: Larousse.

O lé r o n, P. (1983), L'argumentation, Paris: PUF.

Per r a u d e a u, M. (1996), Les méthodes cognitives á l'école, Paris: A. Colin.

P e rri n, L. (1996), L'ironie mise en trope. Du sens des énoncês hyperboliques et ironiques, Paris: Ed. Kimế.

Pè r y - W o od l e y, M.-P. (1993), Les écrits dans l'apprentissage, Paris: Hachette.

Pl a nt in, Ch. (1990), Essais sur l'argumentation. Introduction linguistique à l'étude de la parole argumentative, Paris: Éd. Kimé.

Por t i n e, H. (1981), Un récit dans l'argumentation, Langue Française $n^{\circ}$ 50: 75-89.

R e b o u 1, O. (1984), La rhétorique, Paris: PUF. 
Reboul, O. (1991), Peut-il y avoir une argumentation non rhétorique, Colloque de Cerisy L'argumentation, 107-119. Textes édités par A. Lempereur, Liège: Mardaga.

$\mathrm{R}$ i v e rs, W. Les dix principes de l'apprentissagelenseignement interactif des langues, E.L.A. $\mathrm{n}^{\circ} 77$ : 47-64.

R os s, J. Exploration du style écrit et compêtence communicative, Le Français dans le monde $n^{\circ} 167$ : 42-47.

R o y, G.-R. (1992), Répercussions fächeuses de certaines interférences lecture <-> écriture en enseignement et en apprentissage du français écrit, La lecture et l'écriture. Enseignement et apprentissage: $32-45$.

S p r e n g r - C h a r o ll e s, L. (1980), Le rêsumé de texte, Pratiques n ${ }^{\circ} 26: 59-89$.

Sprenger-Charolles, L. (1982), Quand lire c'est comprendre. Approche linguistique ef psycholinguistique de la lecture, Pratiques $n^{0} 35:$ 7-26.

T a b o u ret-Kelle r, A. (1974), L'écrit. Quelques considérations psychologiques, Le Français dans le monde $\mathrm{n}^{\circ}$ 109: 17-20

Ü b $1 \mathrm{e}$ i s, I. (1982), Apprendre à rédiger: activitês pour un itinéraire, Le Français dans le monde $n^{\circ} 167: 34-41$.

$\mathrm{V}$ a nd e nd or pe, Ch. (1992), Comprendre et interpréter, La Lecture et l'écriture. Enseignement et apprentissage: 150-181, Montréal: Ed. Logiques.

V ig n er, G. (1974), Techniques d'apprentissage de l'argumentation êcrite, Le Français dans le monde $n^{\circ}$ 109: 60-67.

V i g n e r, G. (1982), Élêments pour une pédagogie de l'écrit, Paris: Clé Intern.

V i g n e r, G. (1996), Lire comprendre ou décoder?, Le Français dans le monde n 283: 62-66.

V i g n a u x, G. (1973), Le discours argumenté écrit, Communication n ${ }^{\circ} 20$ : 101-159.

$\mathrm{V}$ i g n a u x, G. (1976), L'Argumentation. Essai d'une logique discursive, Génève: Librairie Droz.

$\mathrm{V}$ i g n a u x, G. (1979), Stratégies dans l'argumentation discursive et modalités d'affirmation du sujet, Sigma $n^{\circ} 4: 33-44$.

W o o d l e y, M.-P., L'écrit en trois dimensions, Le Français dans le monde $n^{\circ}$ 167: 55-69. 